\title{
Approaching the basis set limit for DFT calculations using an environment-adapted minimal basis with perturbation theory: formulation, proof of concept and a pilot implementation
}

\author{
Yuezhi Mao, ${ }^{1}$ Paul R. Horn, ${ }^{1}$ Narbe Mardirossian, ${ }^{1}$ Chris-Kriton Skylaris, ${ }^{2}$ Teresa Head-Gordon, ${ }^{3,4,5}$ \\ and Martin Head-Gordon ${ }^{1,5}$, a) \\ ${ }^{1)}$ Kenneth S. Pitzer Center for Theoretical Chemistry, Department of Chemistry, \\ University of California at Berkeley Berkeley, CA 94720, USA \\ ${ }^{2)}$ School of Chemistry, University of Southampton, Highfield, Southampton, SO17 1BJ, \\ $U K$ \\ ${ }^{3)}$ Department of Chemistry, University of California at Berkeley Berkeley, CA 94720, \\ USA \\ 4) Department of Chemical and Biomolecular Engineering, University of California at Berkeley Berkeley, \\ CA 94720, USA \\ 5) Chemical Science Division, Lawrence Berkeley National Laboratory Berkeley, CA 94720, \\ $U S A$
}

(Dated: 13 March 2016)

Recently developed density functionals have good accuracy for both thermochemistry (TC) and non-covalent interactions (NC) if very large basis sets are used. To approach the basis set limit with potentially lower costs, a new SCF scheme with minimal adaptive basis (MAB) functions variationally optimized on each atomic site is presented, and the desired accuracy can be obtained by applying a perturbative correction (PC). Compared to exact SCF results, using this MAB-SCF (PC) approach with the same target basis set produces $<0.15 \mathrm{kcal} / \mathrm{mol} \mathrm{RMS}$ errors for most of the tested TC datasets, and $<0.1 \mathrm{kcal} / \mathrm{mol}$ for most of the NCs. The performance of density functionals near the basis set limit can be well reproduced with slight discrepancies. With further improvement to its implementation, MAB-SCF (PC) could be a promising substitute for its conventional counterpart to viably approach the basis set limit of modern density functionals.

\section{INTRODUCTION}

Kohn-Sham density functional theory ${ }^{1-3} \quad$ (KSDFT) has become the most widespread electronicstructure method because of its reasonable balance between accuracy and computational cost. Functionals using the generalized gradient approximation (GGA) $)^{4,5}$ are usually regarded as the simplest that can give acceptable accuracy for chemistry. To overcome the plague of self-interaction error, new variables have been introduced, leading to meta-GGA ${ }^{6-8}$, global hybrid $(\mathrm{GH})^{9}$ and rangeseparated hybrid $(\mathrm{RSH})^{10,11}$ functionals. On the other hand, a variety of models have been developed to account for van der Waals (vdW) interactions within $\mathrm{DFT}^{12}$, including the empirical DFT-D methods $^{13-15}$ and nonlocal correlation (NLC) functionals (e.g. vdw-DF-10 ${ }^{16}$, VV10 ${ }^{17}$ ). Most recently, Mardirossian et al. developed two combinatorially designed functionals: $\omega \mathrm{B} 97 \mathrm{X}-\mathrm{V}^{18}$ (RSH+VV10) and B97M-V ${ }^{19}$ (meta-GGA+VV10), which demon-

\footnotetext{
a)Electronic mail: mhg@cchem.berkeley.edu
}

strated impressive accuracy on both thermochemistry (TC) and non-covalent interactions (NC), with an accessible complete basis set (CBS) limit, and low grid sensitivity.

With finite atomic orbital (AO) basis $\operatorname{sets}^{20}$, one prerequisite for attaining such accuracy is to approach the CBS limit. This issue has been carefully investigated $^{21-24}$, but is often neglected in practical applications, as exemplified by the prevalence of the "B3LYP $9,25,26 / 6-31 G(d) "$ model chemistry. A basis set of at least triple- and preferably quadruple$\zeta$ quality is often required by hybrid functionals (e.g. B3LYP) to obtain adequately converged thermochemistry results. Even for the semi-local B97M$\mathrm{V}$ functional, the acceptable alternatives to aug-ccpVQZ ${ }^{27,28}$ (which almost represents the CBS limit) are still of least triple- $\zeta$ quality. Turning to the evaluation of NCs, a similar study on the A24 ${ }^{29}$ and S66 $6^{30,31}$ complexes indicates that augmented triple$\zeta$ basis sets (e.g. aug-cc-pVTZ, def2-TZVPD ${ }^{32}$ ) are in general required by B97M-V to properly converge the binding energies. Their double- $\zeta$ counterparts (e.g. aug-cc-pVDZ, def2-SVPD) should only be carefully used with counterpoise $(\mathrm{CP})$ corrections $^{33}$. For $\omega \mathrm{B} 97 \mathrm{X}-\mathrm{V}$, the requirement on basis set quali- 


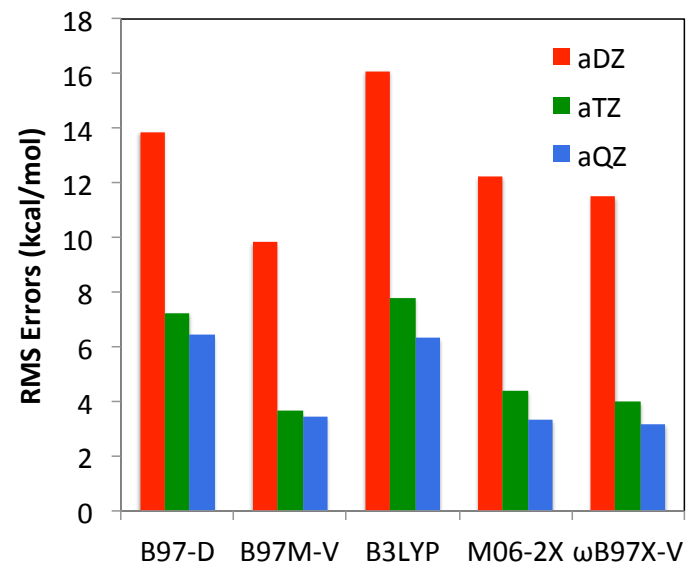

FIG. 1: Basis set dependence of RMS errors for G2 atomization energies against benchmark values from Ref. 35). Data for 5 functionals are collected: B97-D (GGA), B97M-V (meta-GGA), B3LYP (GH), M06-2X $(\mathrm{GH})^{38}$, and $\omega \mathrm{B} 97 \mathrm{X}-\mathrm{V}$ (RSH). Dunning's augmented correlation-consistent basis sets $(\mathrm{D}, \mathrm{T}, \mathrm{Q})$ are used to systematically approach the CBS limit.

ties might be even higher due to the slower basis set convergence of RSH functionals ${ }^{18}$.

Figure 1 demonstrates the basis set convergence of several modern density functionals in terms of their RMSDs for the G2 set $^{34}$ (atomization energies of 148 neutral molecules, whose reference values recomputed by Ref. 35). Apart from B97M-V, using aQZ instead of aTZ for the other 4 functionals reduces their RMSDs by $0.8-1.4 \mathrm{kcal} / \mathrm{mol}$, including the semi-local B97-D ${ }^{14}$ functional (GGA). Using aDZ yields very poor accuracy (usually over 10 $\mathrm{kcal} / \mathrm{mol} \mathrm{RMSDs}$ ) for all these functionals, which defeats the purpose of using state-of-the-art density functionals. One way to tackle this problem is by directly training a functional in a small basis, such as the EDF1 functional ${ }^{36}$, which was parameterized at the $6-31+\mathrm{G}(\mathrm{d})$ level. By relying on a cancellation balance between the functional error and basis set error, their transferability can be limited, and further empirical corrections seem necessary to achieve useful accuracy for relative energies ${ }^{37}$.

Each self-consistent field (SCF) cycle of a KS-DFT calculation involves two computationally significant steps: (1) Fock matrix construction with a given density, and (2) Fock matrix diagonalization to update the density. For fixed system size, the computational cost of the Fock build scales as $\mathcal{O}\left(n^{4}\right)$ with respect to the basis size $(n)$ when conventional AO algorithms are used, and the cost of the diagonalization step scales as $\mathcal{O}\left(n^{3}\right)$. This steep cost increase inhibits large basis sets (e.g. QZ size) from being routinely employed in DFT calculations. The scaling of cost vs. basis size is largely independent of the development of linear scaling (with system size) Fock build algorithms ${ }^{39-45}$ and many diagonalization replacements ${ }^{46,47}$. Moreover, near-complete basis sets are not favored by linear-scaling algorithms, especially when diffuse functions are included, since matrix element sparsity is diminished and the overlap matrix starts to be ill-conditioned, which in turn destroys the sparsity of the density matrix ${ }^{48,49}$.

One successful strategy to make large basis KSDFT calculations more tractable is to compute the full Coulomb $(\mathbf{J})$ and exchange $(\mathbf{K})$ matrices more efficiently by approximating two-electron repulsion integrals (ERIs) with the aid of auxiliary basis functions or grid points. The resolution-of-the-identity (RI) method ${ }^{50-52}$ expands the product of $\mathrm{AO}$ function pairs with a preoptimized auxiliary basis. RI algorithms do not improve the system-size scaling unless local fit regions are applied ${ }^{53,54}$, but they reduce the basis set size scaling from $\mathcal{O}\left(n^{4}\right)$ to $\mathcal{O}\left(n^{3}\right)$. Therefore, state-of-the-art RI algorithms (e.g. MARI-J ${ }^{55}$, occ-RI-K ${ }^{56}$ ) can speed up large basis Fock matrix construction step for small- to medium-sized systems significantly, while retaining numerical accuracy. The diagonalization step is unaffected.

A second successful approach to accelerating large basis calculations is to perform the iterative SCF procedures in a primary (small) basis and then approximate the secondary (target) basis results by utilizing perturbation theory. This idea was intro-

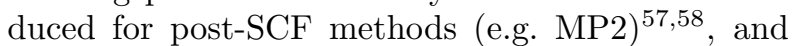
was then developed for SCF methods ${ }^{59-66}$. With a careful choice of primary/secondary basis set pairing, these methods can provide satisfactory accuracy for both $\mathrm{TC}^{59,65,66}$ and $\mathrm{NC}^{63}$ with significantly reduced computational costs (roughly 10 times faster), although system-size scaling remains unchanged. One limitation is the need to develop and validate the basis set pairings ${ }^{63}$, which determines accuracy and speedup. Also, as the secondary basis approaches the CBS limit, the size of the primary basis needed to achieve a given accuracy increases: for instance the optimized primary basis for cc-pVQZ is roughly cc-pVTZ size ${ }^{60}$.

A related approach is the use of small adaptive basis sets. The idea of encoding chemical environment information into atomic/quasiatomic basis functions to understand chemical bonding dates back to early tools ${ }^{67-72}$, as well as more contemporary methods ${ }^{73-78}$. Apart from interpretive purposes, the merits of utilizing small adaptive bases 
in KS-DFT calculations have been recognized with the development of fast (especially linear-scaling) SCF algorithms, leading to renewed interest in the concept of "polarized atomic orbitals" (PAOs), first put forward by Adams in the $1960 \mathrm{~s}^{79,80}$. These adaptive sets usually have very tiny (often minimal) spans, which leads to vastly fewer variational degrees of freedom. In addition, an adaptive basis constructed with spatial confinement contributes to a well-conditioned overlap matrix, which is a property favored by $\mathcal{O}(N)$ scaling methods.

The Adams PAO scheme treats atoms in a molecule as fragments and solves projected equations self-consistently on each of them, which is similar, in spirit, to projected SCF methods using fragment-localized, non-orthogonal MOs to evaluate intermolecular interactions $(\mathrm{SCF}-\mathrm{MI})^{81-83}$. In practice this scheme only works for weakly interacting atoms (e.g. rare-gas clusters) or ionic compounds (e.g. $\mathrm{LiH}, \mathrm{NaCl})^{84}$. Later, the PAO approach was recast to form a minimal atom-centered adaptive basis as an atom-blocked contraction of the secondary basis functions on each atom ${ }^{85}$. The molecular energy is minimized simultaneously with respect to the atom-blocked contraction coefficients and the density matrix in the adaptive basis ${ }^{85}$. The PAO-SCF energy can be improved using perturbation theory ${ }^{86}$, similar to the dual basis approaches discussed above. The minimal rank of the PAO basis and its atomic locality makes it promising for linearscaling algorithms ${ }^{49}$, but the "double" optimization problem is challenging.

Significant progress on tractable adaptive basis schemes for KS-DFT have been made in the condensed matter physics community. Similar to the PAOs, Ozaki and Kino ${ }^{87,88}$ and others ${ }^{89,90}$ used numerical solutions to the atomic Kohn-Sham equations as the secondary basis, and a scheme resembling geometry optimization to obtain the adaptive basis. The CONQUEST program ${ }^{91}$ forms local "support functions" (an adaptive basis) from either functions akin to plane waves $^{92}$ or pseudoatomic orbitals ${ }^{93}$. The ONETEP package ${ }^{94,95}$ forms non-orthogonal generalized Wannier functions (NGWFs) ${ }^{96}$ as the environment-adapted basis, which a linear combination of periodic sinc functions confined in an atom-centered sphere of fixed radius. The NGWFs are efficiently optimized via a preconditioned conjugate-gradient algorithm ${ }^{97}$.

Recently, adaptive basis schemes that do not require the global Hamiltonian or density matrix have been presented. The localized filter diagonalization (LFD) method builds an adaptive basis on-the-fly by contracting the atomic Gaussian functions within a local region, with contraction coefficients determined by diagonalizing a block of the Hamiltonian matrix corresponding to that region ${ }^{98,99}$. This algorithm has also been used to construct multisite local support functions ${ }^{100}$, and the general philosophy has been extended by Lin et al. ${ }^{101}$, including another model with more rigorous optimization ${ }^{102}$. While clearly promising, to our knowledge, the accuracy and performance of these methods on chemical systems have not been systematically assessed yet.

In the present work, we propose an inexpensive version of the PAO method (Sec. II). Instead of energy-optimizing the adaptive basis and density simultaneously, an inexpensive converged SCF solution (density matrix) in a projected reference basis (PRB) is utilized as a reference (Sec. II A). Based on this reference, an atom-centered minimal adaptive basis (MAB) is found by minimizing a judiciously chosen objective function (Sec. IIB), which is computationally inexpensive to evaluate. The converged MAB is then used as the basis set for another SCF calculation, which requires small computational effort as well, and has the promise of providing comparable accuracy to PAO-SCF. Akin to the PAO case, perturbation corrections can be applied to the MAB-SCF energy for improved accuracy (Sec. II E). Details about the pilot implementation and the following benchmark calculations are summarized in Sec. III, and the accuracy of this approximate SCF scheme is assessed on a broad range of TC and $\mathrm{NC}$ datasets in Sec. IV.

\section{THEORY}

The notation used throughout this paper is as follows: $|\omega\rangle$ : generic atomic basis functions; $|\psi\rangle$ : generic molecular orbitals; capital Roman indices $X, Y, \ldots$ : atomic centers; lowercase Greek letters $\mu$, $\nu, \lambda, \ldots$ : secondary (large) AO basis indices; $\alpha, \beta$, $\gamma, \ldots$ : primary (small, contracted) AO basis indices; lowercase Romans $i, j, k, \ldots$ : occupied MO indices; $a, b, c, \ldots$ virtual $\mathrm{MO}$ indices; $p, q, r, \ldots$ generic MO indices. For introducing the MAB optimization scheme, $i, j, \ldots$ are also employed to denote the vectors retained in the MAB subspace, $a, b, \ldots$ for the vectors in MAB's complementary subspace, and $p$, $q, \ldots$ are the generic ones, which is analogous to the partitioning of MO space in SCF.

To concisely show the character of quantities within a nonorthogonal basis, tensorial notation will be used in the derivation, i.e., covariant (subscript) and contravariant (superscript) indices are distinguished, following Ref. 103 and the appendix of 
Ref. 85. For instance, a matrix element denoted by $B_{X \alpha}^{X \mu}$ indicates that matrix $\mathbf{B}$ has rows corresponding to contravariant secondary basis functions and columns corresponding to covariant primary basis functions, and these basis functions belong to the same atomic center $X$. Einstein summation convention is applied for contractions between contravariant and covariant indices, except for summations over different atomic centers, which will be written out explicitly.

\section{A. SCF in a small projected reference basis (PRB)}

The search for the MAB described in Sec. IIB requires an inexpensively calculated reference density matrix in the target basis. A converged SCF solution in a small PRB serves this purpose, where atom-blocked operations ${ }^{62}$ are performed to project the reference basis functions into the space spanned by the target (large) basis:

$$
\begin{aligned}
\left|\omega_{A \alpha}\right\rangle & =\left|\omega_{A \mu}\right\rangle\left(S_{A}^{-1}\right)^{A \mu A \nu}\left(S_{12}\right)_{A \nu A \alpha} \\
& =\left|\omega_{A \mu}\right\rangle B_{A \alpha}^{A \mu} .
\end{aligned}
$$

Here, $\mathbf{S}_{\mathbf{1 2}}$ is the overlap between the (unprojected) $\mathrm{RB}$ and the target basis functions. Throughout this paper, $\mathbf{B}$ will be an atom-blocked matrix containing atomic contraction coefficients of the large basis functions.

Since the reference basis is small (to be specified later) and the target basis is close to the CBS limit, then the atom blocks of $\mathbf{B}$ will be very sparse since the contraction coefficients for the high angular momentum components of the secondary basis all vanish during the projection procedure. At this stage, an SCF calculation is performed in the PRB, by solving the following $\mathrm{KS}$ equations:

$$
\mathcal{F C}=\mathcal{S C} \epsilon,
$$

$\mathcal{F}$ and $\mathcal{S}$ can be transformed from their counterparts in the target basis using the $\mathbf{B}$ matrix:

$$
\mathcal{F}=\mathbf{B}^{T} \mathbf{F B}, \quad \mathcal{S}=\mathbf{B}^{T} \mathbf{S B}
$$

In reverse, the $\mathrm{PRB}$ density matrix, $\mathcal{P}$, can be projected into the target basis via the following transformation:

$$
\tilde{\mathbf{P}}=\mathbf{B} \mathcal{P} \mathbf{B}^{T}
$$

Since the PRB is an exact subset of the secondary basis, no information in $\mathcal{P}$ is lost upon projection into the large basis (Eq. (4)). We call this special property of $\tilde{\mathbf{P}}$ " $B$-representability". The final PRB density matrix $\mathcal{P}$ becomes the reference used in the search for the MAB.

\section{B. Finding the minimal adaptive basis (MAB)}

The previous sub-section employs a basis defined by a fixed atom-blocked transformation (the PRB) and optimizes a density matrix in it. Now our purpose is different. Given the fixed reference density matrix, our goal is to optimize an energy-like function with respect to a variable $\mathbf{B}$ matrix which defines the MAB. (Note: in the following discussion $\mathbf{B}$ denotes the MAB coefficients.) Since a single diagonalization minimizes $\operatorname{Tr}[\mathbf{P F}]$ for a chosen number of electrons ${ }^{60}$ when $\mathbf{F}$ is given, we shall by analogy minimize $\operatorname{Tr}[\tilde{\mathbf{P}} \mathbf{F}]$, where $\tilde{\mathbf{P}}=\mathbf{B} \mathcal{P} \mathbf{B}^{T}$ is a $B$ representable density matrix in the secondary basis that derives from the fixed reference density (denoted as $\mathbf{P}$ here).

However, the MAB has smaller rank than the $\mathrm{PRB}$, and the spaces spanned by them are rather different, so the reference density in the target basis (eq. 4) will not be representable by the MAB. There exist many ways to construct the $B$-representable counterpart of $\mathbf{P}$. We choose to project $\mathbf{P}$ into the space spanned by the MAB first (form $\mathcal{P}$ ), and then transform it back into the large basis:

$$
\begin{aligned}
\mathcal{P} & =\left(\boldsymbol{\sigma}^{-1}\right) \mathbf{B}^{T} \operatorname{SPSB}\left(\boldsymbol{\sigma}^{-1}\right), \\
\tilde{\mathbf{P}} & =\mathbf{B} \mathcal{P} \mathbf{B}^{T} \\
& =\mathbf{B}\left(\boldsymbol{\sigma}^{-1}\right) \mathbf{B}^{T} \operatorname{SPSB}\left(\boldsymbol{\sigma}^{-1}\right) \mathbf{B}^{T} .
\end{aligned}
$$

Recognizing that the projector into the MAB space is:

$$
\mathbf{R}=\mathbf{B}\left(\boldsymbol{\sigma}^{-1}\right) \mathbf{B}^{T}
$$

then our objective function becomes:

$$
E=\operatorname{Tr}[\mathbf{R S P S R F}]
$$

We note that the $B$-representable density matrix $\tilde{\mathbf{P}}$ usually does not contain exactly the right electron count. While the exact $N_{\text {elec }}$ is given by $\operatorname{Tr}[\mathbf{P S}]$, based on the idempotency of $\mathbf{R}$, we have

$$
\begin{aligned}
\operatorname{Tr}[\tilde{\mathbf{P S}}] & =\operatorname{Tr}[\mathbf{R S P S R S}] \\
& =\operatorname{Tr}[\mathbf{R S P S}] \neq \operatorname{Tr}[\mathbf{P S}],
\end{aligned}
$$

The inequality arises because the reference density matrix $\mathbf{P}$ is usually not $B$-representable.

Gradient-based optimization can locate the optimal $\mathbf{B}$ as the minimizer of Eq. (8). The initial guess for the MAB (and its complementary basis, denoted by $\mathbf{V}$ ), is obtained by diagonalizing atomic blocks of the reference density matrix, appropriately transformed $^{71}$ as $\mathbf{P}_{\mathbf{A}}^{\prime}=\mathbf{X}_{\mathbf{A}}^{\mathbf{T}} \mathbf{S}_{\mathbf{A}} \mathbf{P}_{\mathbf{A}} \mathbf{S}_{\mathbf{A}} \mathbf{X}_{\mathbf{A}}$, where $\mathbf{S}_{\mathbf{A}}$ 
is the overlap matrix of the secondary basis functions on $A$, and $\mathbf{X}_{\mathbf{A}}$ is the canonical orthogonalizer for them. Denoting $\mathbf{U}_{\mathbf{A}}$ as the eigenvectors of $\mathbf{P}_{\mathbf{A}}^{\prime}$, the initial $\mathbf{B}$ and $\mathbf{V}$ are set to:

$$
\begin{aligned}
\left(B_{A}^{\mathrm{init}}\right)_{A i}^{A \mu} & =\left(X_{A}\right)_{A p}^{A \mu}\left(U_{A}\right)_{A i}^{A p} \\
\left(V_{A}^{\mathrm{init}}\right)_{A a}^{A \mu} & =\left(X_{A}\right)_{A p}^{A \mu}\left(U_{A}\right)_{A a}^{A p}
\end{aligned}
$$

The partitioning of the Hilbert space can be represented symbolically as

$$
\begin{aligned}
\mathbb{I}_{\mathbf{A}} & =\mathbf{X}_{\mathbf{A}}=\mathbf{B}_{\mathbf{A}} \oplus \mathbf{V}_{\mathbf{A}} \\
\mathbb{I} & =\bigoplus_{A=1}^{N_{A}} \mathbb{I}_{\mathbf{A}}
\end{aligned}
$$

Since the MAB functions (and the complementary ones) are constructed by on-site contractions of the secondary basis, the variables that parameterize the MAB are intra-atomic orbital rotations. Akin to Ref. 49, a single on-block unitary transform is parameterized by the exponential of an antisymmetric matrix $^{104}$, which ensures that the updated atomic orbitals stay on the same manifold:

$$
[B(\theta)]_{X i}^{X \mu}=C_{X r}^{X \mu} \exp \left(\theta_{X i}^{X r}\right),
$$

where $C_{X_{r}}^{X_{\mu}}$ denotes the union of the MAB and the complementary functions on atom $X$. To enforce antisymmetry of $\boldsymbol{\theta}$, it is further parameterized by $\boldsymbol{\Delta}$ which actually contains all the independent variables:

$$
\begin{aligned}
\theta_{X i}^{X r} & =\left[\Delta^{X r X s}-\left(\Delta^{\dagger}\right)^{X r X s}\right] \sigma_{X s X i} \\
& =\left[\Delta^{X r X s}-\Delta^{X s X r}\right] \sigma_{X s X i} .
\end{aligned}
$$

The desired gradient, evaluated at $\boldsymbol{\Delta}=\mathbf{0}$ is

$$
\begin{aligned}
\frac{\partial E}{\partial \Delta^{z_{p q}}} & =\sum_{X, Y} \frac{\partial E}{\partial R^{X \mu Y \nu}} \frac{\partial R^{X \mu Y \nu}}{\partial \Delta^{z_{p Z q}}} \\
& =\sum_{X, Y}(S P S R F+F R S P S)_{Y \nu X \mu} \frac{\partial R^{X \mu Y \nu}}{\partial \Delta^{z_{p q} Z^{\prime}}}
\end{aligned}
$$

$E$ is invariant with respect to orbital rotations within the MAB space $(p=i, q=j)$, or within the space of complementary excluded vectors $(p=a, q=$ $b$ ), as these rotations leave $\mathbf{R}$ unchanged. Therefore, the non-zero gradient comes only from variations of $\Delta^{Z i z a}$. Using the identities

$$
\frac{\partial B_{X j}^{X \mu}}{\partial \Delta^{Z i Z a}}=-V_{X a}^{X \mu} \delta_{Z}^{X} \sigma_{X i X j}
$$

and

$$
\frac{\partial\left(\boldsymbol{\sigma}^{-1}\right)}{\partial \boldsymbol{\Delta}}=-\left(\boldsymbol{\sigma}^{-1}\right) \frac{\partial \boldsymbol{\sigma}}{\partial \boldsymbol{\Delta}}\left(\boldsymbol{\sigma}^{-1}\right)
$$

the desired gradient expression is given by

$$
\frac{\partial E}{\partial \Delta^{Z_{i Z a}}}=-2 \sigma_{Z_{i Z_{j}}}\left[\left(\sigma^{-1}\right) B^{T} G(I-R S)\right]_{Z_{\mu}}^{Z_{j}} V_{Z_{a}}^{z_{\mu}},
$$

where, for brevity, $\mathbf{G}=\partial E / \partial \mathbf{R}$ as defined in Eq. 14. More details about the derivation of Eq. (17) is provided in Appendix A.

Once the gradient at the current position is computed, the optimization algorithm will generate a new step $(\boldsymbol{\Delta})$ based on it (and the previous gradients and steps). The equations for the exponential transformation were derived by Ref. 104. The update for the MAB can be represented as

$$
\mathbf{B}^{(n)}=\mathbf{B}^{(n-1)}\left(\begin{array}{c}
\mathbf{U} \cos \boldsymbol{p}^{1 / 2} \mathbf{U}^{\mathbf{T}} \\
-\boldsymbol{\Delta}^{\dagger} \mathbf{U} \boldsymbol{p}^{1 / 2} \sin \boldsymbol{p}^{1 / 2} \mathbf{U}^{\mathbf{T}}
\end{array}\right) .
$$

$\mathbf{U}$ and $\boldsymbol{p}$ stand for eigenvectors and eigenvalues of the matrix quantity $\boldsymbol{\Delta} \boldsymbol{\Delta}^{\dagger}$, respectively, and note that the unitary transformations are atom-blocked operations. When the iterative optimization converges, B represents a minimal basis energetically adapted to the chemical environment described by the reference density matrix (from PRB-SCF). Figure 2 illustrates the MAB optimization procedure. Finally, we note that for unrestricted cases, the MABs for $\alpha$ and $\beta$ electrons are optimized separately (they are completely decoupled), using the same objective function form.

With the MAB defined, a converged SCF solution can be obtained in this basis. The SCF energy in the fixed MAB will be an approximation to the energy evaluated by PAO-SCF, which directly minimizes the SCF energy with respect to the generators of the MAB as well as the variables defining the density matrix. These two approaches will be compared in Sec. IV A.

\section{Modified definition of the minimal adaptive basis}

The size of a minimal basis only depends on the principal quantum number of the atom's valence shell, since a complete set of angular momentum functions are needed to fulfill the requirement of spatial isotropy. This definition usually works very well, but there are two types of exceptions. First, in some cases, the standard rank of the minimal basis includes redundant functions. For example, the minimal basis of lithium $(n=2)$ consists of 5 functions, 


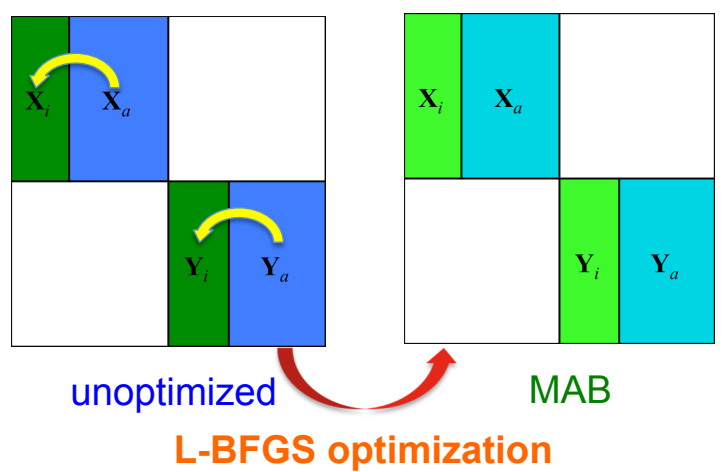

FIG. 2: Illustration of the MAB optimization procedure. $X$ and $Y$ are two distinct atom blocks. $X_{i}$, $Y_{i}$ represent the MAB functions, and $X_{a}, Y_{a}$ are the excluded basis functions.

although only two of them are required to describe its $1 s^{2} 2 s^{1}$ configuration. The same applies to some other electron-deficient species like cations and radicals. The presence of redundant functions causes difficulties in converging the MAB optimization procedure. Second, in some cases, the standard rank of the minimal basis is too small to accurately describe the bonding. Examples include some hypervalent molecules (e.g. $\mathrm{SO}_{3}, \mathrm{ClF}_{3}$, etc), and, occasionally, molecular anions. In such cases, the standard rank of the MAB will lead to larger errors in molecular properties, which can be greatly reduced if a certain number of additional MAB functions are judiciously added to the appropriate atomic centers.

In both cases, we can adjust the rank of the MAB appropriately based on information that is already available from the initial PRB-SCF calculation. The resulting procedure, shown in Algorithm 1, can either truncate or augment the MAB dimension on each atom. The number of significant eigenvalues $\left(N_{\text {Sig }}\right)$ for each atom is set to the number of eigenvalues of $\mathbf{P}_{\mathbf{A}}^{\prime}$ that are above a first threshold (thresh 1 has a default value of 0.01 ). The MAB dimension will be reduced to $N_{S i g}$ if that is smaller than a minimal basis $\left(N_{\min }\right)$. On the other hand, when $N_{\text {min }}<N_{\text {Sig }}$, the algorithm expands the MAB dimension by the number of eigenvalues beyond $N_{\text {min }}$ that satisfy $E_{A}(i) / E_{A}\left(N_{\text {min }}\right)>$ thresh2 (thresh2 has a default value of 0.02 , i.e., eigenvalues that are larger than $E_{A}\left(N_{\min }\right) / 50$ will be included), which will allow a lower optimized MAB-SCF energy.

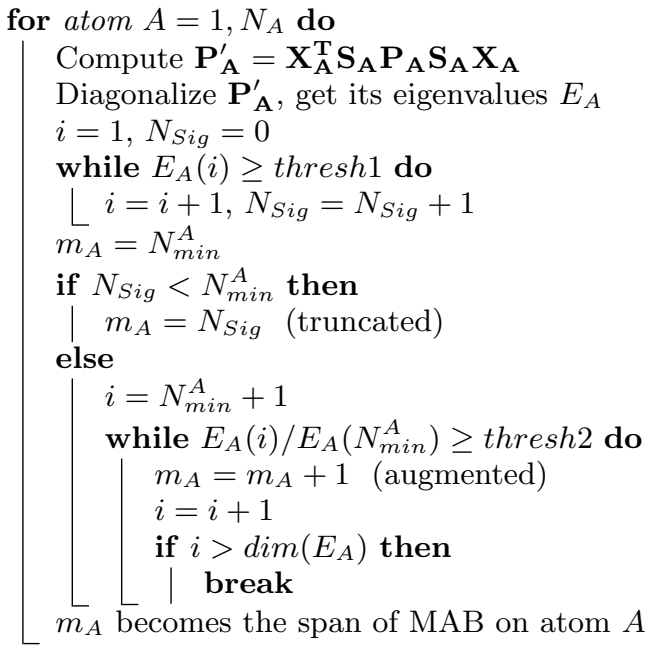

Algorithm 1: Algorithm that flexibly adjusts (truncates or augments) the size of the MAB for special systems, based on quantities already computed when generating the initial guess for the MAB optimization.

\section{Modified MAB objective function}

The objective function given by Eq. (8) can be rewritten as follows:

$$
\begin{aligned}
E & =\operatorname{Tr}\left[\mathbf{R S C}_{o} \mathbf{C}_{o}^{T} \mathbf{S R F}\right] \\
& =\operatorname{Tr}\left[\mathbf{C}_{o}^{T} \mathbf{S R F R S C}_{o}\right] \\
& =\operatorname{Tr}\left[\tilde{\mathbf{C}}_{o}^{T} \mathbf{F} \tilde{\mathbf{C}}_{o}\right],
\end{aligned}
$$

where $\tilde{\mathbf{C}}_{o}=\mathbf{R S C}_{o}$ represents the PRB-optimized occupied MOs after projection into the MAB space. For stable species, the energies of occupied MOs should all be negative, and thus minimizing E corresponds to retaining as many of the bound electrons as possible.

A disaster occurs in the MAB optimization if an occupied MO has a positive energy, because minimization will result in loss of those electrons. With inexact functionals, this occasionally happens for anions. For example, the energy of the $2 p$ orbitals in $\mathrm{F}^{-}$is $0.001 \mathrm{Eh}$ with the B3LYP functional (hybrid), and $0.056 \mathrm{Eh}$ with the B97-D functional (pure). With these functionals, the resulting value of $\operatorname{Tr}[\mathbf{R S P S}]$ (number of electrons captured by $\tilde{\mathbf{P}}$ ) is close to 2 when the MAB is optimized, which indicates that the six $2 p$ electrons are missing! This is completely unphysical, and causes the SCF energy computed with the MAB to be qualitatively incorrect. 
Such difficulties can be avoided by modifying the eigenvalue structure of $\mathbf{F}$ to ensure that all occupied levels are negative. This can be done by applying a uniform shift to all the eigenvalues:

$$
\mathbf{F}^{\prime}=\mathbf{F}-\lambda \mathbf{S}
$$

using $\mathbf{C}^{\mathbf{T}} \mathbf{S C}=\mathbf{I}$. The shift, $\lambda$, is set to be:

$$
\lambda=\varepsilon(\mathrm{HOMO})+0.75[\varepsilon(\mathrm{LUMO})-\varepsilon(\mathrm{HOMO})],
$$

so that the zero energy lies between the PRB-SCF HOMO and LUMO. Replacing $\mathbf{F}$ with $\mathbf{F}^{\prime}$ in Eq. (8) gives a modified objective function for the MAB:

$$
\begin{aligned}
E & =\operatorname{Tr}[\mathbf{R S P S R}(\mathbf{F}-\lambda \mathbf{S})] \\
& =\operatorname{Tr}[\mathbf{R S P S R F}]-\lambda \operatorname{Tr}[\mathbf{R S P S}]
\end{aligned}
$$

When $\lambda>0$, the new term resembles a penalty for losing electrons, which can be made explicit by adding an additional constant, $\lambda N_{\text {elec }}$, to the RHS:

$$
E=\operatorname{Tr}[\mathbf{R S P S R F}]+\lambda\left(N_{\text {elec }}-\operatorname{Tr}[\mathbf{R S P S}]\right) .
$$

\section{E. Perturbation correction schemes}

Based on the data presented in Ref. 85 and 86, a significant difference exists between PAO-SCF and exact SCF results. To reduce this gap, computationally inexpensive correction schemes based on perturbation theory are useful. Analogous to the dual-basis method, the converged MAB-SCF solution serves as the primary basis reference, and the contribution of non-Brillouin singles to the secondorder perturbative (PT2) energy correction is given by $^{59}$

$$
\delta E=\operatorname{Tr}\left[\mathbf{F}_{O V}^{(1)} \mathbf{T}_{V O}^{(1)}\right] .
$$

Here $\mathbf{F}$ denotes the Fock matrix built upon the MAB-SCF density projected into the secondary basis: $\mathbf{F}=\mathbf{F}(\tilde{\mathbf{P}})$. The first-order $T$-amplitude satisfies the following equation:

$$
\mathbf{F}_{V O}^{(1)}+\mathbf{F}_{V V}^{(0)} \mathbf{T}_{V O}^{(1)}-\mathbf{T}_{V O}^{(1)} \mathbf{F}_{O O}^{(0)}=\mathbf{0}_{V O}^{(1)} .
$$

In the pseudo-canonicalized MO basis (obtained by diagonalizing $\mathbf{F}_{O O}$ and $\mathbf{F}_{V V}$ separately, see Appendix C), Eq. (25) reduces to a simpler form:

$$
T_{a i}^{(1)}=-F_{a i}^{(1)} /\left(\epsilon_{a}^{(0)}-\epsilon_{i}^{(0)}\right) .
$$

Correspondingly, the perturbative energy lowering becomes

$$
\delta E=-\sum_{i a}\left|F_{a i}^{(1)}\right|^{2} /\left(\epsilon_{a}^{(0)}-\epsilon_{i}^{(0)}\right),
$$

which can be interpreted as an energy-weighted steepest descent (approximate Newton) step ${ }^{59,86}$. Alternatively, other corrections that involve a full diagonalization of the Fock matrix can be applied, such as the aforementioned DBDF (only slightly different from PT2) and DFPC methods. The latter performs a single update of the density matrix in the secondary basis (by diagonalizing $\mathbf{F}$ ), and then recomputes the full SCF energy based on that (the result will thus be variational).

\section{COMPUTATIONAL DETAILS}

To summarize, the full MAB-SCF scheme consists of three stages:

1. Project the target basis onto a small basis to form the PRB; perform the first-stage SCF calculation within its span (PRB-SCF), and collect $\mathbf{P}$ and $\mathbf{F}(\mathbf{P})$ at convergence.

2. Based on the reference provided by PRB-SCF, optimize the MAB for each atom using gradientbased algorithms; perform the second-stage SCF calculation with the optimized MAB (MAB$\mathrm{SCF})$.

3. On top of the MAB-SCF solution, apply a perturbation correction to further approach the exact $\mathrm{SCF}$ result in the target basis.

A pilot implementation of this method is accomplished in a development version of the Q-Chem 4.3 package $^{105}$. A preconditioned limited-memory BFGS (L-BFGS) algorithm ${ }^{106,107}$ is implemented for solving the MAB optimization problem efficiently. The inverted on-diagonal blocks of the Hessian matrix for the objective function (second derivatives with regard to orbital rotations on the same atomic site) are employed as the preconditioner of the LBFGS algorithm. In most scenarios, this preconditioning strategy leads to convergence of the MAB optimization in a reasonable number of iterations $\left(10^{1} \sim 10^{2}\right)$, while the additional cost for evaluating the preconditioner is moderate. More details about the preconditioned L-BFGS algorithm and the evaluation of the on-diagonal blocks of the Hessian are provided in Appendix B.

In the current implementation, all the density matrix updates are computed by diagonalizing $\mathcal{F}$ (Fock matrix in the dimension of PRB or MAB), and the only diagonalization in the large basis dimension $\left(N_{v} \times N_{v}\right.$ to be exact) is required by the perturbation correction. However, to obtain $\mathcal{F}$, it still requires the 
contraction of the ERI tensor in the secondary basis with the $B$-representable density matrix for the time being, as

$$
\begin{aligned}
\left(F_{\alpha}\right)_{\mu \nu}= & h_{\mu \nu}+(\mu \nu \mid \lambda \sigma) \tilde{P}_{\mathrm{tot}}^{\lambda \sigma}-\kappa(\mu \sigma \mid \lambda \nu) \tilde{P}_{\alpha}^{\lambda \sigma} \\
& +\left[V_{x c}\left(\tilde{P}_{\alpha}, \tilde{P}_{\beta}\right)\right]_{\mu \nu} .
\end{aligned}
$$

for the $\alpha$ electrons ( $\kappa$ is the proportion of exact exchange in the employed functional). Then $\mathbf{F}$ is transformed back into the primary basis through Eq. 3. This choice is actually less efficient, because quantities in the primary basis, like $\mathcal{P}^{\alpha \beta}, \mathcal{C}_{i}^{\alpha}$, can be directly utilized, which significantly reduces the dimension of the contraction. Therefore, with our preliminary implementation, we will focus on validating the accuracy of MAB-SCF (PC) in this work, and the potential efficient implementation of this method will be briefly discussed in Sec. V.

All the results for TC and NC datasets are generated with the KS-DFT routines in Q-Chem 4.3 as well. A $(75,302)$ grid (75 radial shells with 302 Lebedev points in each) is used for all employed exchange-correlation (XC) functionals, and the SG-1 grid $^{108}$ is used for the VV10 NLC functional. Unless otherwise noted, $6-31+\mathrm{G}(\mathrm{d})$ is used as the reference basis in PRB-SCF, and the optimization of the MAB converges to $10^{-6}$ while all the SCF calculations converge to $10^{-8}$. To determine the appropriate dimension of the MAB, the default values of thresh 1 and thresh 2 adopted by Algorithm 1 are set to 0.01 and 0.02 , respectively. We note that in this work, the "adding vector" strategy is by default turned off and only utilized for user-specified hypervalent molecules.

\section{RESULTS AND DISCUSSIONS}

\section{A. Preliminary tests on G2 set}

\section{Comparison with PAO-SCF}

We start investigating the accuracy of our method by performing a series of preliminary tests on the G2 $\mathrm{set}^{34}$. To demonstrate the quality of the optimized $\mathrm{MAB}$, the performance of MAB-SCF on the G2 set is compared to $\mathrm{PAO}-\mathrm{SCF}$, since the latter gives the limiting behavior of an atom-centered minimal basis. For the reasons discussed in Sec. II C, the minimal basis models (including MAB and $\mathrm{PAO}$ ) are not sufficient for describing the hypervalent molecules. Thus, we designate the molecules centered by $\mathrm{Al}-$ $\mathrm{Cl}$ and coordinated by highly electronegative atoms

\begin{tabular}{ccccccc}
\hline \multicolumn{4}{c}{ B97-D $^{\mathrm{a}}$} & \multicolumn{1}{c}{ B97M-V } \\
\hline SCF energies & \multicolumn{1}{c}{ B3LYP $^{\mathrm{C}}$} \\
MAB & PAO & MAB & PAO & MAB & PAO \\
MAX & 25.11 & 25.48 & 26.70 & 26.96 & 24.39 & 24.85 \\
RMSD & 7.07 & 7.27 & 8.06 & 8.07 & 7.05 & 7.18 \\
MSE & 5.59 & 5.72 & 6.54 & 6.49 & 5.62 & 5.66 \\
\hline With PT2 correction \\
MAB PAO & MAB & PAO & MAB & PAO \\
MAX & -0.26 & -0.14 & -0.14 & -0.08 & 0.84 & 0.91 \\
RMSD & 0.06 & 0.03 & 0.03 & 0.02 & 0.18 & 0.20 \\
MSE & -0.02 & 0.00 & -0.02 & -0.01 & 0.14 & 0.15 \\
\hline
\end{tabular}

${ }^{a}$ Convergence failures: $\cdot \mathrm{CCH}$

${ }^{\text {b }}$ Convergence failures: $\mathrm{SO}_{2}, \mathrm{ClF}_{3}, \cdot \mathrm{SH}$

${ }^{\mathrm{c}}$ Convergence failures: $\mathrm{NaCl}$

TABLE I: Summary of the PAO-SCF and MAB-SCF results (with and without PT2 correction) on the "pruned" G2 set. 6-31+G(d) is used as the reference

basis in PRB-SCF, while 6-31++G(d,p) is used for the atomic energy of $\mathrm{H}$ (not for $\mathrm{H}$ atoms in molecules).

Errors (in $\mathrm{kcal} / \mathrm{mol}$ ) are evaluated with respect to the conventional SCF results (aQZ is the target basis). Molecules that fail to converge their PAOs (listed in the footnotes) are excluded when evaluating the statistical errors.

(e.g. $\mathrm{O}, \mathrm{F}, \mathrm{Cl}$ ) as hypervalent ones, including $\mathrm{SO}$, $\mathrm{ClO}, \mathrm{SO}_{2}, \mathrm{AlF}_{3}, \mathrm{AlCl}_{3}, \mathrm{SiF}_{4}, \mathrm{SiCl}_{4}, \mathrm{PF}_{3}, \mathrm{ClF}_{3}$, and $\left(\mathrm{CH}_{3}\right)_{2} \mathrm{SO}$, and exclude them from the test set temporarily. The results for this "pruned" G2 set (138 molecules) computed with three distinct functionals are collected in Table I (aug-cc-pVQZ is the target basis). To make it a fair comparison, molecules that fail to converge their PAOs (listed in the table footnotes) are also excluded when reporting the statistical errors. At the SCF level (without PT2 correction), PAO- and MAB-SCF show similar accuracy with respect to the exact SCF results for all the three tested functionals, and in general the MAB-SCF results are slightly better as a result of error cancellation (PAO-SCF is exact for atomic energies). Applying the PT2 correction significantly reduces the errors for both schemes. For the two tested pure functionals (B97-D and B97M-V), the RMSDs of MAB+PT2 are smaller than $0.1 \mathrm{kcal} / \mathrm{mol}$ $(\sim 0.05 \mathrm{kcal} / \mathrm{mol})$, and they are close to those of $\mathrm{PAO}+\mathrm{PT} 2$; for B3LYP, the RMSDs of both schemes remarkably increase, which may indicate the diminished effectiveness of PT2 when hybrid functionals are used. Nevertheless, we notice that the performance of $\mathrm{MAB}+\mathrm{PT} 2$ is rather similar to that of $\mathrm{PAO}+\mathrm{PT} 2$ (the former slightly outperforms here). 
To accurately compute the energies of those hypervalent molecules, we increase the dimensions of their MAB based on Algorithm 1. The MAB+PT2 results computed thereof are compared with those using the standard MAB and PAO model. The improvement on their atomization energies are exhibited in Table II. For a majority of these molecules $\left(\mathrm{ClO}, \mathrm{SiF}_{4}, \mathrm{SiCl}_{4}, \mathrm{PF}_{3},\left(\mathrm{CH}_{3}\right)_{2} \mathrm{SO}\right.$, and presumably $\mathrm{SO}_{2}$ and $\mathrm{ClF}_{3}$ ), the errors are reduced by over 10 times by using the "adding vector" strategy, compared to those utilizing the standard PAO or MAB models. The demonstrated inadequacy of the conventional minimal basis models can be used to characterize molecular hypervalency. Among these molecules, species like $\mathrm{SiF}_{4}, \mathrm{PF}_{3}$ do not formally violate the "octet" rule, which indicates that molecular hypervalency may exist beyond its traditional definition. On the other hand, $\mathrm{AlCl}_{3}$ and $\mathrm{AlF}_{3}$ seem not to be typical hypervalent species, because the minimal basis models do not entirely break down as on the other molecules listed in Table II (PAO+PT2 achieves almost the same accuracy as the modified MAB+PT2 approach). Combining these specially treated hypervalent molecules with other 138 molecules computed with the standard MAB model, the overall RMSD for the G2 set is $0.033 \mathrm{kcal} / \mathrm{mol}$, which is only minimally different from the result for the "pruned" G2 set (with B97M$\mathrm{V}$ ), while the largely increased standard $\mathrm{MAB}+\mathrm{PT} 2$ and $\mathrm{PAO}+\mathrm{PT} 2$ errors when hypervalent molecules are included further reveals the necessity of modifying their $\mathrm{MAB}$ dimensions. In the following tests performed on G2, unless otherwise specified, statistics evaluated with all the molecules will be reported with the hypervalent ones separately treated.

Table III shows how the dimensions of the MAB are increased on the central atoms of these hypervalent molecules after applying Algorithm 1. According to the rightmost column, the modified MAB function counts are usually close to those of a minimal basis with one set of $d$ (polarization) functions. One interesting fact is that although $\mathrm{AlF}_{3}$ and $\mathrm{AlCl}_{3}$ do not show typical hypervalent character according to Table II, in both the $\mathrm{Al}$ atom tends to add more vectors into the $\mathrm{MAB}$, although they only make small differences energetically. However, we notice that the situation becomes different when B3LYP is used. Significant improvement on the $\mathrm{AlCl}_{3}$ molecule is achieved by using the modified MAB model, in terms of the errors vs. exact SCF results (modified $\mathrm{MAB}+\mathrm{PT} 2: 0.03 \mathrm{kcal} / \mathrm{mol} ; \mathrm{PAO}+\mathrm{PT} 2$ : $0.21 \mathrm{kcal} / \mathrm{mol}$ ). A similar situation occurs on the $\mathrm{SO}$ molecule as well (modified MAB+PT2: 0.08 $\mathrm{kcal} / \mathrm{mol} ; \mathrm{PAO}+\mathrm{PT} 2: 0.46 \mathrm{kcal} / \mathrm{mol})$. We see the

\begin{tabular}{|c|c|c|c|}
\hline & $\begin{array}{c}\text { MAB+PT2 } \\
\text { (normal) }\end{array}$ & $\begin{array}{c}\text { MAB+PT2 } \\
\text { (add_vec) }\end{array}$ & $\mathrm{PAO}+\mathrm{PT} 2$ \\
\hline $\mathrm{SO}$ & 0.049 & 0.022 & 0.085 \\
\hline $\mathrm{ClO}$ & -0.182 & 0.010 & -0.120 \\
\hline $\mathrm{SO}_{2}$ & -0.990 & 0.001 & $\mathrm{~N} / \mathrm{A}$ \\
\hline $\mathrm{AlF}_{3}$ & -0.030 & 0.002 & 0.002 \\
\hline $\mathrm{AlCl}_{3}$ & 0.050 & -0.016 & -0.017 \\
\hline $\mathrm{SiF}_{4}$ & -0.214 & -0.007 & -0.268 \\
\hline $\mathrm{SiCl}_{4}$ & -0.445 & -0.009 & -0.544 \\
\hline $\mathrm{PF}_{3}$ & -0.720 & 0.009 & -0.544 \\
\hline $\mathrm{ClF}_{3}$ & -1.857 & -0.020 & $\mathrm{~N} / \mathrm{A}$ \\
\hline $\mathrm{SO}\left(\mathrm{CH}_{3}\right)_{2}$ & -1.173 & -0.026 & -1.061 \\
\hline \multicolumn{4}{|c|}{ G2 statistics (all molecules) } \\
\hline MAX & -1.857 & -0.141 & -1.061 \\
\hline RMSD & 0.238 & 0.033 & 0.114 \\
\hline MSE & -0.058 & -0.017 & -0.024 \\
\hline
\end{tabular}

TABLE II: Errors (in $\mathrm{kcal} / \mathrm{mol}$ ) for the atomization energies of the hypervalent molecules in the G2 set. Results of unmodified $\mathrm{MAB}+\mathrm{PT} 2, \mathrm{MAB}+\mathrm{PT} 2$ with the "adding vector" strategy, and $\mathrm{PAO}+\mathrm{PT} 2$ are compared. All the calculations are performed with the B97M-V functional, using aug-cc-pVQZ as the large basis. The corresponding statistical errors for the full G2 set (including these molecules) are also reported.

\begin{tabular}{cccc}
\hline Molecule & $\begin{array}{c}\text { Central } \\
\text { atom }\end{array}$ & dim (MAB) & $\operatorname{dim}(\mathrm{MAB})$ \\
(original) & (add_vec) \\
\hline $\mathrm{AlF}_{3}$ & $\mathrm{Al}$ & 9 & 16 \\
$\mathrm{AlCl}_{3}$ & $\mathrm{Al}$ & 9 & 13 \\
\hline $\mathrm{SiF}_{4}$ & $\mathrm{Si}$ & 9 & 15 \\
$\mathrm{SiCl}_{4}$ & $\mathrm{Si}$ & 9 & 15 \\
\hline $\mathrm{PF}_{3}$ & $\mathrm{P}$ & 9 & 14 \\
\hline $\mathrm{SO}$ & $\mathrm{S}$ & 9 & 13 \\
$\mathrm{SO}_{2}$ & $\mathrm{~S}$ & 9 & 13 \\
$\left(\mathrm{CH}_{3}\right)_{2} \mathrm{SO}$ & $\mathrm{S}$ & 9 & 12 \\
\hline $\mathrm{ClO}$ & $\mathrm{Cl}$ & 9 & 12 \\
$\mathrm{ClF}_{3}$ & $\mathrm{Cl}$ & 9 & 14 \\
\hline
\end{tabular}

TABLE III: Modifications of the MAB dimensions on the central atoms of the studied hypervalent molecules in the G2 set, after the "adding vector" policy is applied. The number of the MAB functions for the coordinating atoms are not necessarily remaining the same.

merits of Algorithm 1 from these scenarios since it consistently generates small errors regardless of the choice of functionals.

The efficiency for the two schemes to obtain the optimized adaptive basis is also investigated. In general, the optimization problem for the MAB is con- 


\begin{tabular}{|c|c|c|c|c|c|}
\hline \multirow[b]{2}{*}{ Molecules } & \multicolumn{2}{|c|}{ Num of opt steps } & \multicolumn{3}{|c|}{ Num of Fock builds } \\
\hline & $\mathrm{PAO}$ & MAB & $\overline{\mathrm{PAO}}$ & $\overline{\mathrm{PRB}}$ & MAB \\
\hline $\mathrm{CH}$ & 277 & 93 & 277 & 27 & 6 \\
\hline $\mathrm{Na}_{2}$ & 1219 & 23 & 1219 & 7 & 3 \\
\hline $\mathrm{Si}_{2}$ & 145 & 20 & 145 & 16 & 22 \\
\hline $\mathrm{NaCl}$ & 3208 & 47 & 3208 & 9 & 5 \\
\hline $\mathrm{SO}_{2}$ & $\mathrm{~N} / \mathrm{A}$ & 212 & $\mathrm{~N} / \mathrm{A}$ & 11 & 8 \\
\hline $\mathrm{COS}$ & 148 & 146 & 148 & 10 & 7 \\
\hline $\mathrm{ClF}_{3}$ & $\mathrm{~N} / \mathrm{A}$ & 83 & $\mathrm{~N} / \mathrm{A}$ & 12 & 8 \\
\hline $\mathrm{C}_{2} \mathrm{Cl}_{4}$ & 614 & 80 & 614 & 9 & 5 \\
\hline $\mathrm{C}_{4} \mathrm{H}_{6}$ (2-butyne) & 490 & 149 & 490 & 10 & 7 \\
\hline $\mathrm{C}_{2} \mathrm{H}_{4} \mathrm{O}$ & 109 & 111 & 109 & 9 & 6 \\
\hline $\mathrm{SH}$ & $\mathrm{N} / \mathrm{A}$ & 60 & $\mathrm{~N} / \mathrm{A}$ & 48 & 8 \\
\hline $\mathrm{CH}_{3} \mathrm{CH}_{2} \mathrm{O}$ & 450 & 156 & 450 & 44 & 9 \\
\hline
\end{tabular}

TABLE IV: Molecules in the G2 set that require over 100 iterations to converge their PAOs. Number of iterative optimization steps and Fock builds required to reach the convergence of $\mathrm{PAO}-\mathrm{SCF}$ and $\mathrm{MAB}-\mathrm{SCF}$ are compared. For several radicals $\left(\mathrm{CH}, \cdot \mathrm{SH}, \mathrm{CH}_{3} \mathrm{CH}_{2} \mathrm{O} \cdot\right)$, considerably larger number of iterations are required by the PRB-SCF step because GDM algorithm ${ }^{109}$ is employed to circumvent the unstable SCF solutions.

siderably easier to converge compared to the "double" optimization required by PAO-SCF. No convergence failure for $\mathrm{MAB}$ is detected for the entire G2 set with all three tested functionals. Moreover, in contrast with the formulation of PAO-SCF, the search for the MAB is decoupled with any density matrix optimization, and thus its iteration counts will not directly affect the required number of SCF cycles. This is extremely important because of the much more significant cost per iteration for the latter. Table IV lists molecules in the G2 set that require over 100 iterations to converge their PAOs (with B97M-V/aQZ). Besides those that fail to converge, for molecules such as $\mathrm{NaCl}$, the PAO-SCF scheme also runs into major convergence difficulties, which in turn requires an enormous number of Fock matrix constructions. The MAB scheme, on the other hand, attains the optimized adaptive basis in less number of iterations for most of these molecules. In the scenarios as the $\mathrm{COS}$ and $\mathrm{C}_{2} \mathrm{H}_{4} \mathrm{O}$ (oxirane) molecules, although the iteration counts for the two schemes are very close, MAB-SCF is still far more efficient because much fewer Fock builds are required. Therefore, MAB-SCF can be regarded as a more feasible adaptive basis SCF scheme compared to PAO$\mathrm{SCF}$, with comparable accuracy remained.

\section{Functional and basis set transferability}

So far the formulation of MAB-SCF does not involve any functional-specific parameter. However, according to the results in Table I, the performance of $\mathrm{MAB}+\mathrm{PT} 2$ is not completely functionalindependent. A clear difference exists between using pure and hybrid functionals. Therefore, it will be meaningful to investigate the performance of this method when different flavors of density functionals are employed.

Table V collects the RMSDs for the MAB $+\mathrm{PT} 2$ approach (vs. exact SCF) with various density functionals evaluated on the G2 set (aQZ is used as the target basis). The first seven studied functionals do not contain exact exchange, including three GGAs (B97-D, BLYP ${ }^{25,26}, \mathrm{PBE}^{5}$ ) and four meta-GGAs (TPSS $^{8}$, MGGA_MS1 ${ }^{110}$, M06-L ${ }^{111}$, B97M-V); and the remaining six are hybrid functionals, including TPSSh $(10 \%)^{112}$, B3LYP $(20 \%)$, PBE0 $(25 \%)^{113}$, M06-2X (54\%), $\omega$ B97X-D (RSH) ${ }^{114}$, and $\omega$ B97X$\mathrm{V}(\mathrm{RSH})$ ("\%" denotes the proportion of exact exchange). Results of MAB-SCF with another perturbation correction scheme, DFPC, are also presented for comparison. In general, the $\mathrm{MAB}+\mathrm{PT} 2$ scheme demonstrates good accuracy for the tested GGA and meta-GGA functionals, while the RMSDs computed by MAB + DFPC are roughly twice as large, which indicates that PT2 is the preferable perturbation correction scheme for pure functionals. M06-L seems to be the only outlier whose RMSD is over 0.1 $\mathrm{kcal} / \mathrm{mol}$, which is related to the unbalanced performance of $\mathrm{MAB}+\mathrm{PT} 2$ on atoms and molecules with this functional (the MSE for the absolute atomic energies is $+0.05 \mathrm{kcal} / \mathrm{mol}$, while that for the molecules is $-0.09 \mathrm{kcal} / \mathrm{mol}$ ).

The situation turns out to be different for the hybrid functionals. The PT2 approach undershoots the exact SCF energy in general, and the size of errors grows with the increased amount of exact exchange, which results in unsatisfactory accuracy (RMSD > $0.2 \mathrm{kcal} / \mathrm{mol}$ ) for functionals that contain more exact exchange than B3LYP. The MAB+DFPC approach, on the other hand, manifests comparatively more robust performance across all the tested hybrid functionals (RMSDs are around $0.1 \mathrm{kcal} / \mathrm{mol}$ ), and it consistently outperforms the undershooting PT2 approach. For the two RSH functionals, the results of $\mathrm{MAB}+\mathrm{DFPC}$ are 5-6 times more accurate than $\mathrm{MAB}+\mathrm{PT} 2$. Based on these results, we currently suggest that DFPC be used as the correction to MAB-SCF when hybrid functionals are employed, at the expense of one more Fock build in the secondary basis, while $\mathrm{MAB}+\mathrm{PT} 2$ is more favorable for pure functionals. Further study will be required to improve the accuracy of MAB-based methods for hybrid functionals without involving additional cost. In practice, different basis sets can be employed 


\begin{tabular}{ccc}
\hline Functionals & MAB+PT2 & MAB+DFPC \\
\hline B97-D & 0.053 & 0.115 \\
BLYP & 0.074 & 0.148 \\
PBE & 0.065 & 0.132 \\
TPSS & 0.055 & 0.119 \\
MGGA_MS1 & 0.042 & 0.108 \\
M06-L & 0.144 & 0.156 \\
B97M-V & 0.033 & 0.079 \\
\hline TPSSh & 0.099 & 0.086 \\
B3LYP & 0.181 & 0.083 \\
PBE0 & 0.220 & 0.066 \\
M06-2X & 0.458 & 0.076 \\
$\omega$ B97X-D & 0.645 & 0.119 \\
$\omega$ B97X-V & 0.668 & 0.133 \\
\hline
\end{tabular}

TABLE V: RMSDs (in kcal/mol) of MAB-SCF with two different perturbation correction schemes (PT2 and DFPC) for G2 set. Different pure (B97-D, BLYP, PBE, TPSS,MGGA_MS1, M06-L, B97M-V) and hybrid

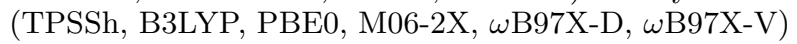
functionals are investigated. aQZ is employed as the target large basis for all the calculations.

for KS-DFT calculations, based on the balance between accuracy and efficiency, available benchmark results from references, and also user preference. With the B97M-V functional, we assess the performance of MAB+PT2 with several widely used basis sets, including those in Dunning's correlation-consistent series (aTZ, QZ, aQZ) ${ }^{27,28}$, Jensen's polarization-consistent series (apc-2, pc3, apc-3) $)^{115-117}$, and the Karlsruhe def2 series (TZVPPD, QZVPP, QZVPPD) ${ }^{32}$. The popular "large Pople" basis set $6-311++\mathrm{G}(3 \mathrm{df}, 3 \mathrm{pd})^{118,119}$ is also included. All the tested basis sets are of or above augmented triple- $\zeta$ quality, because the goal of our method is to approach the results of modern density functionals near the CBS limit.

The RMSDs for the G2 set using different target basis sets are displayed in Figure 3. The errors are below $0.1 \mathrm{kcal} / \mathrm{mol}$ for most of the tested basis sets, which indicates the excellent transferability in general. The best performance is achieved by two quadruple- $\zeta$ basis sets with diffuse functions, aQZ and QZVPPD. And for their unaugmented counterparts, QZ and QZVPP, the RMSDs are slightly larger, while the accuracy is still considered to be satisfactory. For ordinary bonded interactions, the diffuse functions usually have no major impact on the accuracy of evaluated energetics, so the compatibility of MAB+PT2 with these unaugmented basis sets is also meaningful. The largest RMS errors are produced by Jensen's pc-3 and aug-pc-3 basis sets, mostly due to the poor performance of $\mathrm{MAB}+\mathrm{PT} 2$ for the Li atom when these target basis sets are used. The RMSDs are reduced to 0.063 (pc-3) and 0.059 (apc-3) $\mathrm{kcal} / \mathrm{mol}$ if we exclude three Li-containing molecules $\left(\mathrm{Li}_{2}, \mathrm{LiF}\right.$, and $\left.\mathrm{LiH}\right)$ from the $\mathrm{G} 2$ set, which are actually similar to the results of the quadruple$\zeta$ sets in Dunning and Karlsruhe series. The outlier might be related to the poor compatibility of pc-3/apc-3 with the employed reference basis, 6$31+\mathrm{G}(\mathrm{d})$, for the Li atom. Without considering about the computational cost, aQZ and QZVPPD should be recommended as the target basis, for the purpose of approaching the true CBS limit and also the top accuracy of our method.

\section{Basis set convergence of density functionals with MAB}

Ultimately, MAB-SCF with a certain perturbation correction scheme (MAB-SCF (PC)) is supposed to replace conventional SCF in KS-DFT calculations when large $\mathrm{AO}$ basis sets that are close to the CBS limit are employed. It is important to see how MAB-SCF (PC) approaches the basis set limit when we increase the size of the target basis. For that purpose, we extend Figure 4 (which motivates this work) with the functional RMSDs evaluated by $\mathrm{MAB}+\mathrm{PT} 2$ (for B97-D, B97M-V) and $\mathrm{MAB}+\mathrm{DFPC}$ (for B3LYP, M06-2X, $\omega$ B97X-V), as demonstrated by Figure 4 . The performance of these functionals at different basis set levels is reproduced by MABSCF $(\mathrm{PC})$ with minimal differences, and the trend for each functional to reach the basis set convergence in a stepwise way $(\mathrm{D}, \mathrm{T}, \mathrm{Q})$ is also precisely reflected. At the aQZ level, the largest difference between MAB-SCF (PC) and exact SCF results is only about $0.03 \mathrm{kcal} / \mathrm{mol}$ (for B97-D and B3LYP), which is below $1 \%$ of the functional RMSDs and thus considered negligible. More appreciable differences between these two sets of results exist at the aDZ level (the largest gap is $0.26 \mathrm{kcal} / \mathrm{mol}$ for $\omega \mathrm{B} 97 \mathrm{X}$ $\mathrm{V} / \mathrm{aDZ}$ ), while at the same time, it is barely meaningful for a double- $\zeta$ basis set to be the target of MAB-SCF (PC). These results, in general, are also consistent with the conclusions of the basis set transferability test presented above (Figure 3 ). 


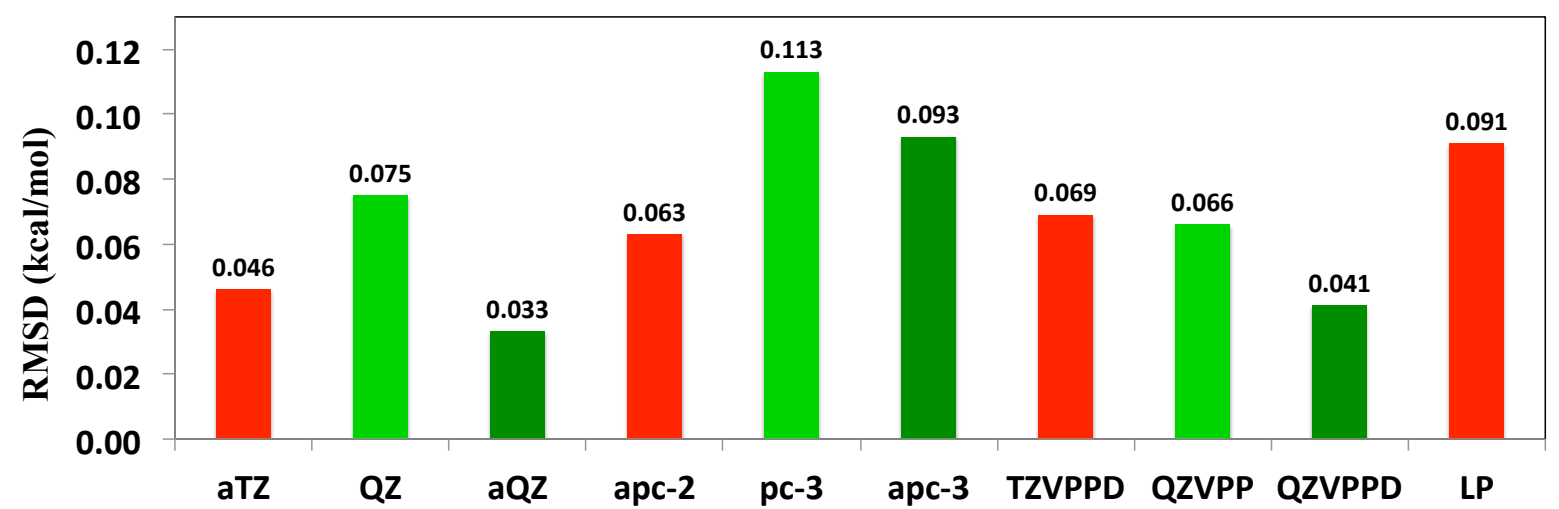

FIG. 3: Assessment of the performance of MAB+PT2 with 10 basis sets. RMSDs (vs. exact SCF) for the G2 set calculated with the B97M-V functional are reported. Different colors are utilized for different basis categories: augmented triple- $\zeta$ (red), unaugmented quadruple- $\zeta$ (light green), and augmented quadruple- $\zeta$ (dark green).

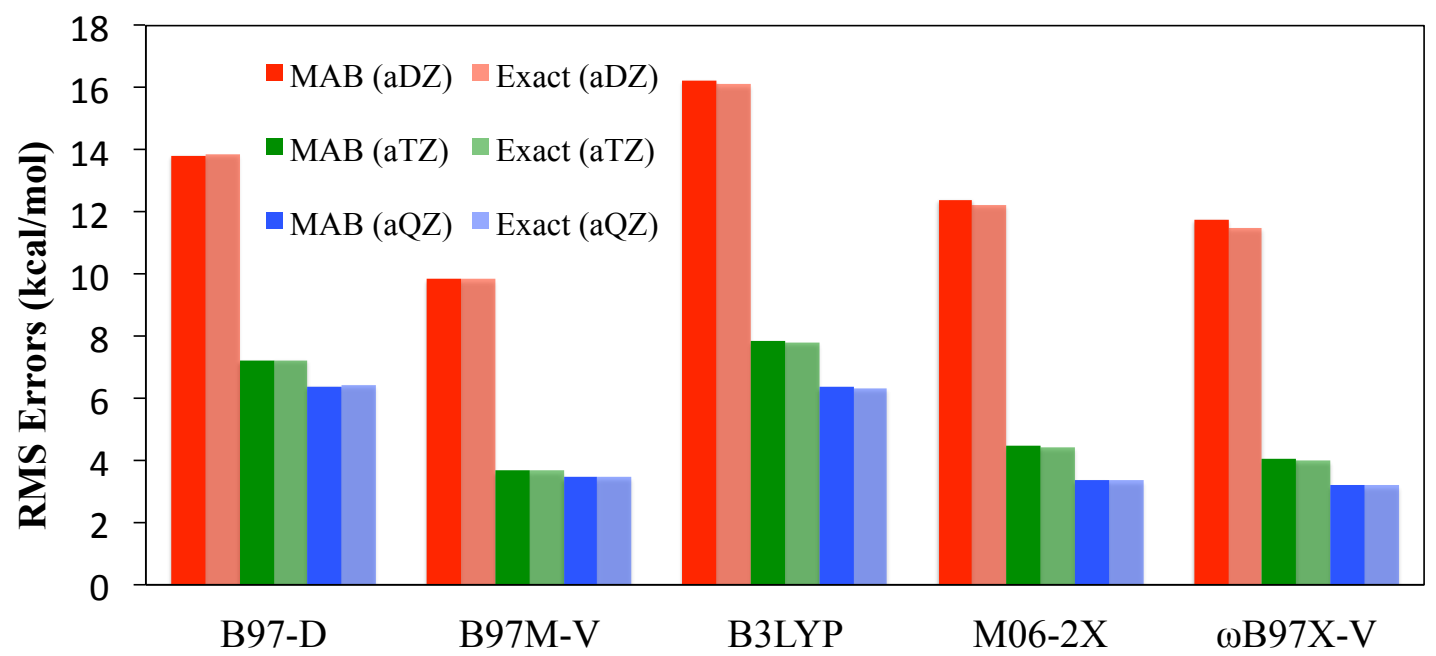

FIG. 4: Basis set convergence of the functional RMSDs for the G2 set evaluated by MAB-based methods (solid) and conventional SCF (translucent). The reference values are provided by Ref. 35. Dunning's augmented correlation-consistent basis sets are utilized to approach the CBS limit (D: red, T: green, Q: blue). PT2 and DFPC correction schemes are applied on top of the MAB-SCF results of local (B97-D, B97M-V) and hybrid (B3LYP, M06-2X, $\omega$ B97X-V) functionals, respectively.

\section{B. Additional TC and NC tests}

\section{Thermochemistry}

Since all the tests so far are performed on the G2 atomization energies, it will be necessary to assess how the performance of MAB-SCF (PC) transfers to other thermochemistry (TC) datasets. Three density functionals (B97-D, B97M-V, B3LYP) are employed to examine the accuracy of MAB-SCF (PC), and aQZ is used as the target basis for all the following TC tests. The choice of perturbation correction schemes is consistent with that in Figure 4.

First we discuss the results for the W4-11 dataset $^{120}$, which consists of about 1000 data points, including 99 bond dissociation energies (BDE99), 707 heavy-atom transfer reaction energies (HAT707), 20 isomerization energies (ISO20), 13 nucleophilic substitution reaction energies (SN13), and 140 total atomization energies (TAE140). Among the molecules requested for generating all the data points, the following 13 are separately treated as hypervalent molecules: $\mathrm{AlF}_{3}, \mathrm{AlCl}_{3}, \mathrm{SiF}_{4}, \mathrm{P}_{4}, \mathrm{SO}$, $\mathrm{SO}_{2}, \mathrm{SO}_{3}, \mathrm{~S}_{2} \mathrm{O}, \mathrm{S}_{2}, \mathrm{~S}_{3}, \mathrm{~S}_{4}, \mathrm{ClO}$, and $\mathrm{OClO}$ (see 


\begin{tabular}{|c|c|c|c|}
\hline & B97-D & B97M- & B3LYP \\
\hline BDE99 & 0.061 & 0.038 & 0.070 \\
\hline HAT707 & 0.101 & 0.061 & 0.100 \\
\hline ISO20 & 0.068 & 0.048 & 0.085 \\
\hline SN13 & 0.034 & 0.018 & 0.053 \\
\hline TAE140 & 0.071 & 0.046 & 0.096 \\
\hline Overall & 0.093 & 0.057 & 0.096 \\
\hline
\end{tabular}

TABLE VI: RMSDs (in kcal/mol) of MAB-SCF (PC) for the W4-11 dataset (vs. exact SCF) evaluated by three different functionals. PT2 is applied for B97-D and B97M-V while DFPC is used for B3LYP. aug-cc-pVQZ is the target large basis.

the previous discussion for the G2 set). Table VI contains the RMSDs of the MAB-SCF (PC) approach (vs. exact SCF) for W4-11, where different categories of TC have been separated. The overall performance is similar to that for the G2 set, which demonstrates the transferability of the accuracy of MAB-SCF (PC) to this more comprehensive TC dataset. Taking B97M-V as an example, the smallest and largest RMSDs of MAB+PT2 (vs. exact SCF) are obtained on SN13 $(0.02 \mathrm{kcal} / \mathrm{mol})$ and HAT707 $(0.06 \mathrm{kcal} / \mathrm{mol})$, respectively, while the corresponding functional RMSDs (vs. W4 reference) are $1.39 \mathrm{kcal} / \mathrm{mol}$ and $3.90 \mathrm{kcal} / \mathrm{mol}$. Therefore, the errors caused by replacing conventional SCF with MAB-SCF (PC) are usually smaller than the size of typical functional errors by one or two orders of magnitude. A more straightforward comparison is provided by Figure 5: for B97M-V, the functional RMSDs computed via the MAB+PT2 approach show almost no difference compared to those by normal SCF method.

Besides W4-11, the performance of MABSCF (PC) is also examined on other categories of TC datasets, including: adiabatic ionization potentials and electron affinities (21 for each) of atoms and small molecules (G21IP and G21EA) ${ }^{121,122}, 38$ hydrogen transfer and 38 heavy-atom transfer barrier heights (NHTBH $38^{123}$ and HTBH38 ${ }^{124}$ ), and 14 alkane isomerization energies (Pentane14 $4^{125}$ ). The computational details are identical to the previous calculations for W4-11, except for the anions in G21EA, HTBH38 and NHTBH38, where the modification of MAB objective function introduced in Sec. IID is applied to avoid unphysical results. The RMSDs of MAB-SCF (PC) are presented in Table VII. According to these results, HTBH38

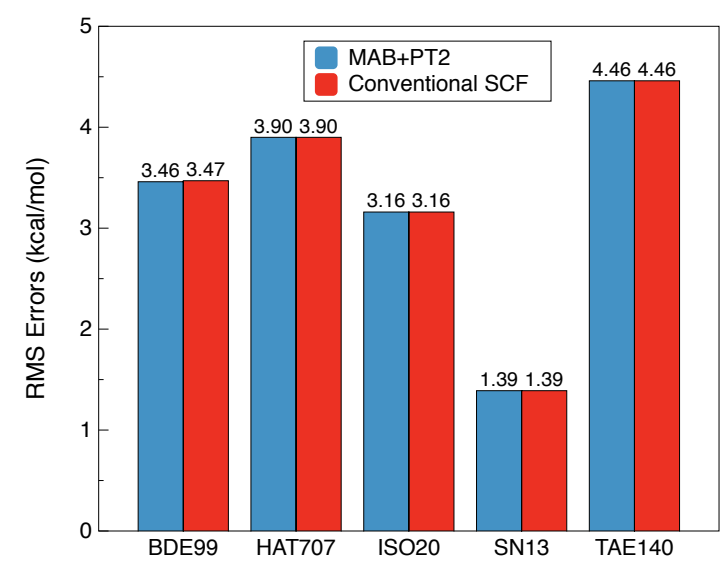

FIG. 5: B97M-V's RMSDs for W4-11 (vs. reference values) evaluated by MAB+PT2 (blue) and conventional SCF (red). Very close accuracy is obtained by these two SCF schemes at the B97M-V/aug-cc-pVQZ level of theory.

and Pentane14 are relatively easier cases for MABSCF (PC) to approximate the exact SCF result; and for G21IP and NHTBH38, the size of the RMSDs is similar to that for the W4-11 subsets (e.g. BDE99, SN13, etc.). The largest RMSDs for thermochemistry so far appears on the G21EA dataset. Although none of the results are qualitatively incorrect with the modified MAB objective function, there still exist several molecular anions whose absolute energies evaluated by MAB-SCF $(\mathrm{PC})$ are rather unsatisfactory: $\mathrm{NO}^{-}, \mathrm{PO}^{-}, \mathrm{O}_{2}^{-}$, and $\mathrm{S}_{2}^{-}$. Based on the discussions in Sec. II C, the "adding vector" strategy may also be applied onto these electronabundant species, and the absolute energies of these molecules computed thereof are significantly improved, as shown in TableVIII. With these 4 molecular anions specially treated, the RMSDs for the G21EA dataset are also recalculated and the results are also presented in Table VII, which turn out to be more comparable to the RMSDs for other categories of TC.

\section{Non-covalent interactions}

One of the most important features of modern density functionals is their capability to account for non-covalent interactions (NC). Therefore, we proceed by examining the performance of MABSCF (PC) on several representative NC datasets, including: A24 (24 small NC complexes) ${ }^{29}$, S22 (22 small to medium $\mathrm{NC}$ complexes at the equi- 


\begin{tabular}{cccc}
\hline \multicolumn{5}{c}{ B97-D } & B97M-V & B3LYP \\
\hline G21IP & 0.050 & 0.036 & 0.040 \\
\hline \multirow{2}{*}{ G21EA } & 0.598 & 0.412 & 0.455 \\
& $(0.101)$ & $(0.027)$ & $(0.078)$ \\
\hline HTBH38 & 0.016 & 0.035 & 0.031 \\
NHTBH38 & 0.099 & 0.071 & 0.118 \\
\hline Pentane14 & 0.005 & 0.007 & 0.002 \\
\hline
\end{tabular}

TABLE VII: RMSDs (in $\mathrm{kcal} / \mathrm{mol}$ ) of MAB-SCF (PC) for TC datasets other than W4-11 (G21IP, G21EA, HTBH38, NHTBH38, Pentane14).

The computational details are the same as in Table VI except for the modification of MAB scheme for the involved anions. The numbers in parentheses are the RMS errors for G21EA after applying the "adding vector" strategy for $\mathrm{NO}^{-}, \mathrm{PO}^{-}, \mathrm{O}_{2}^{-}$, and $\mathrm{S}_{2}^{-}$.

\begin{tabular}{lccccccc}
\hline & \multicolumn{3}{c}{ Normal } & & \multicolumn{3}{c}{ Add_Vec } \\
\cline { 2 - 3 } \cline { 6 - 7 } \cline { 6 - 7 } & B97-D & B97M-V & B3LYP & & B97-D & B97M-V & B3LYP \\
\hline NO $^{-}$ & -2.36 & -1.63 & 1.60 & & -0.13 & -0.06 & 0.10 \\
PO $^{-}$ & -0.52 & -0.55 & 0.92 & & -0.02 & -0.02 & 0.17 \\
$\mathrm{O}_{2}^{-}$ & -2.04 & -1.25 & 1.66 & & -0.01 & -0.02 & 0.07 \\
$\mathrm{~S}_{2}^{-}$ & -0.41 & -0.16 & 0.23 & & -0.01 & 0.02 & 0.02 \\
\hline
\end{tabular}

TABLE VIII: Errors in kcal/mol (vs. exact SCF) for the absolute energies of 4 molecular anions $\left(\mathrm{NO}^{-}\right.$, $\mathrm{PO}^{-}, \mathrm{O}_{2}^{-}$, and $\mathrm{S}_{2}^{-}$) evaluated by normal MAB-SCF (PC) ("Normal") and that with the "adding vector" strategy ("Add_Vec"). Other computational details are the same as in Table VII.

librium geometries) ${ }^{126,127}$, HB15 (15 ionic hydrogen bond interactions) ${ }^{128}$, H2O6Bind8 (binding energies of 8 configurations of water hexamers $)^{129,130}$, and FmH2O10 (binding energies of 10 configurations of $\left.\mathrm{F}^{-}\left(\mathrm{H}_{2} \mathrm{O}\right)_{10}{ }^{129,130}\right)$. Since many of these interactions have smaller magnitudes compared to the common energy scale in TC datasets (the strongest interaction in $\mathrm{S} 22$ is about $-20 \mathrm{kcal} / \mathrm{mol}$ ), and modern density functionals with some certain dispersion correction schemes are able to achieve very small errors for them (e.g. B97M-V's overall RMSD for NC is 0.22 $\left.\mathrm{kcal} / \mathrm{mol}^{19}\right)$, higher requirement on accuracy will be imposed on the MAB-based approaches to match the exact SCF results.

As in the previous discussions on $\mathrm{TC}$, three density functionals (B97-D, B97M-V, B3LYP-D3) are employed to assess the performance of MAB$\mathrm{SCF}(\mathrm{PC})$ on these NC datasets. Based on the arguments in Sec. I, to avoid the necessity of using counterpoise (CP) corrections, we choose QZVPPD as the target basis which has less functions than aQZ but generates even smaller BSSE. Table IX contains the resulting RMSDs (vs. exact SCF) for these NC datasets. Very small errors are yielded by utilizing the MAB-SCF (PC) approximation for the tested dimer binding energies (including data points in A24, S22, and HB15), with all the three functionals. Its good performance on A24 and S22 validates the accuracy of this method in evaluating the most common non-covalent interactions as a replacement of conventional SCF. Larger errors appear on the cluster binding energies (H2O6Bind8, FmH2O10), due the larger magnitude of these interactions in general (H2O6Bind8: -40 to $-50 \mathrm{kcal} / \mathrm{mol}$; FmH2O10: about $-170 \mathrm{kcal} / \mathrm{mol}$ ) and the uniformity of their interaction types (systematic errors accumulate in one single direction through all the data points). One outlier is the RMSD for FmH2O10 when B3LYP-D3 is employed, which is caused by the less accurate MAB-DFPC result for $\mathrm{F}^{-}$with that functional (the error vs. exact $\mathrm{SCF}$ is $0.292 \mathrm{kcal} / \mathrm{mol}$ ). As an alternative, MAB+PT2 generates an RMSD about 3 times smaller for that specific dataset, although statistically it is less preferable for hybrid functionals.

The accurate description of these non-covalent interactions by B97M-V can also be reproduced using MAB-SCF (PC), and the resulting RMS errors (vs. reference) are compared with the exact SCF results in Figure 6. Only minimal differences exist between two sets of RMSDs for the dimer binding energies, while the monotonic deviations of the MAB-SCF (PC) results for each single data point contribute to more pronounced differences for the clusters. Nevertheless, even for H2O6Bind8 where the largest deviation occurs, the RMSD vs. exact SCF results $(0.085 \mathrm{kcal} / \mathrm{mol})$ is only $0.1-0.2 \%$ of the magnitude of the corresponding binding energies. In terms of the accurate evaluation of relative energies, the errors caused by using MAB-SCF (PC) are still negligible.

Finally we are going to examine the accuracy of MAB-SCF (PC) on large non-covalent complexes. The recently proposed L7 data set ${ }^{131}$ is employed, which is comprised with seven large complexes that are mostly stabilized by dispersion interactions (the abbreviations for their names simply follow Ref. 131): stacked circumcoronene-adenine dimer (C3A), stacked circumcoronene with a Watson-Crick G-C base pair (C3GC), parallel displaced coronene dimer (C2C2PD), stacked Watson-Crick G-C base pairs (GCGC), stacked guanine trimer (GGG), parallel stacked octadecane dimer $(\mathrm{CBH})$, and phenylalanine residue trimer (PHE). Due to the tremendous computational effort required by these systems, calculations are performed with two pure function- 


\begin{tabular}{lccc}
\hline & B97-D & B97M-V & B3LYP-D3 \\
\hline A24 & 0.009 & 0.005 & 0.009 \\
S22 & 0.015 & 0.023 & 0.025 \\
HB15 & 0.021 & 0.027 & 0.023 \\
H2O6Bind8 & 0.061 & 0.085 & 0.118 \\
FmH2O10 & 0.078 & 0.026 & $0.350(0.121)$ \\
\hline
\end{tabular}

TABLE IX: RMS errors (in $\mathrm{kcal} / \mathrm{mol}$ ) of MAB-SCF (PC) for 5 NC datasets: A24, S22, HB15, H2O6Bind8, and FmH2O10. Data points computed by conventional SCF using the def2-QZVPPD basis set (noCP) provide the reference values. The number in the parenthesis shows the RMSD of the alternative $\mathrm{MAB}+\mathrm{PT} 2$ method.

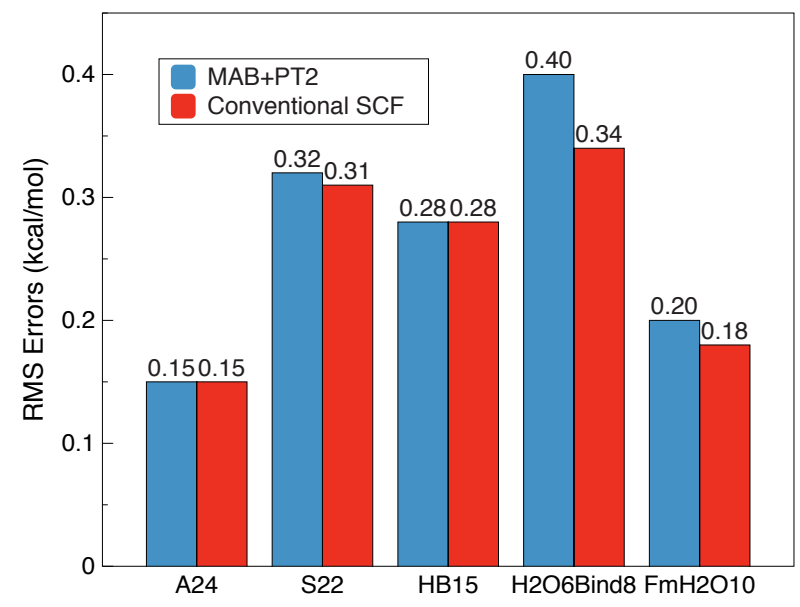

FIG. 6: B97M-V's RMSDs for $5 \mathrm{NC}$ datasets (vs. reference values) evaluated by $\mathrm{MAB}+\mathrm{PT} 2$ (blue) and conventional SCF (red), respectively. def2-QZVPPD is employed as the basis set without using counterpoise corrections.

als (B97-D, B97M-V) using aTZ (which is smaller than QZVPPD) as the target basis. Table $\mathrm{X}$ contains the absolute errors and unsigned percent errors for the 7 binding energies (measured against exact $\mathrm{SCF}$ results). For most of them, the errors resulting from the MAB-SCF (PC) approach are much smaller than $1 \%$ of the magnitude of their binding energies, which indicates the satisfactory accuracy of this approximate SCF scheme for the non-covalent interactions in these large systems. The only exception is the guanine trimer (GGG), which is mostly due to the very weak binding $(-2.33 \mathrm{kcal} / \mathrm{mol}$ by B97M$\mathrm{V} / \mathrm{aTZ}$ ) of that complex. Due to the lack of reliable reference values, the comparison of functional RMSDs evaluated by MAB-SCF (PC) and conven-

\begin{tabular}{lccccc}
\hline & \multicolumn{2}{c}{ B97-D } & & \multicolumn{2}{c}{ B97M-V } \\
\cline { 2 - 3 } \cline { 5 - 6 } Complexe & Error & Error (\%) & & Error & Error (\%) \\
\hline C3A & 0.009 & $0.05 \%$ & & -0.022 & $0.13 \%$ \\
C3GC & 0.008 & $0.03 \%$ & & -0.079 & $0.26 \%$ \\
C2C2PD & 0.011 & $0.05 \%$ & & -0.012 & $0.06 \%$ \\
GCGC & 0.006 & $0.04 \%$ & & -0.053 & $0.34 \%$ \\
GGG & -0.046 & $1.80 \%$ & & -0.063 & $2.63 \%$ \\
CBH & 0.005 & $0.03 \%$ & & 0.003 & $-0.02 \%$ \\
PBH & 0.021 & $0.09 \%$ & & -0.005 & $0.02 \%$ \\
\hline
\end{tabular}

TABLE X: Absolute errors (in kcal $/ \mathrm{mol}$ ) and unsigned percent errors of the MAB-SCF (PC) approach (vs. exact SCF results) for the L7 binding energies. The performance is assessed with using two pure density functionals with corrections for dispersion

(B97-D, B97M-V). aug-cc-pVTZ is the target basis.

tional SCF is currently not available for the L7 dataset.

\section{DISCUSSION AND FUTURE WORK}

So far we have assessed the accuracy of MABSCF (PC) on a broad range of TC and NC datasets. Although using the MAB will inevitably bring an additional source of error to KS-DFT calculations, the overall accuracy of this new approximate SCF method seems to be encouraging. When approaching the basis set limit, the functional RMSDs for several representative TC and NC datasets (G2, W4-11, S22) evaluated by MAB-SCF (PC) show very small differences compared to the exact SCF results, as demonstrated in Figures 4, 5, and 6. In particular, we would like to point out that "B97M-V/aQZ (or QZVPPD)/MAB+PT2" could be a promising model chemistry, because of B97M-V's great accuracy for both $\mathrm{TC}$ and $\mathrm{NC}$, moderate computational cost as a semi-local functional, and high compatibility with the MAB+PT2 approach.

Challenges still exist in applying MAB-SCF (PC) for hybrid functionals. Although the resulting accuracy of $\mathrm{MAB}+\mathrm{DFPC}$ is satisfactory in most of the tested cases, one extra Fock build upon the density matrix in the large basis (not a $B$-representable one) is required, which should be preferably avoided (the reason will be elucidated below); on the other hand, the accuracy of MAB+DFPC slightly degrades for RSH functionals as well (Table V) despite its remarkable advantage over $\mathrm{MAB}+\mathrm{PT} 2$. We also need to note that hypervalent molecules are treated separately in this work by modifying the MAB dimensions in a semi-automated way (on/off of the 
"adding vector" approach is user-specified), because our current choice of the algorithm and the related thresholds focuses on attaining the desired accuracy for those hypervalent molecules exclusively, which might therefore be excessive for the other molecules: convergence difficulties of the MAB optimization can take place when unnecessary vectors are added, and the cost of MAB-SCF gets increased as well. Method that treats all the molecules more consistently could be devised with further investigations.

Although this paper is mainly about the formulation MAB-SCF (PC) and the validation of its accuracy, we should recall that the ultimate goal of this approximate SCF method is to achieve the accuracy of modern density functionals at the basis set limit with lower costs, and eventually it serves as an inexpensive substitute for conventional SCF. In contrast with normal KS-DFT calculations within large basis sets, our method solves for the SCF energy within much lower degrees of freedom (the dimension of PRB or MAB). The cost of a single density matrix update, if achieved by diagonalizing the Fock matrix, can be reduced by up to a factor of $(n / m)^{3}$, where $n$ is the size of the secondary basis, and $m$ is the size of the PRB or MAB. For example, given a model system $\left(\mathrm{CH}_{2}\right)_{\mathrm{n}}$, there will be 129 basis functions per $-\mathrm{CH}_{2}-$ unit if QZVPPD is employed as the target basis, which is roughly 6 times as large as the PRB (22 functions/unit), and about 18 times as the MAB (7 functions/unit). Therefore, the prefactor of the computational cost of this cubic scaling step is significantly reduced in our scheme. One single diagonalization of the large-basis $\mathbf{F}$, however, is still required by performing the perturbation correction (either PT2 or DFPC). New approaches (perturbative or variational) that utilize a more compact representation of the virtual MOs are under development to further reduce the computational prefactor of that step.

Alternatively, $\mathcal{O}(N)$ scaling electronic structure methods can be easily applied to the MAB-SCF step, since the overlap matrix of the MAB is extremely well-conditioned, as demonstrated by Table XI. The diagonalization-free density matrix update algorithms introduced by Ref. 49 can be adopted due to the similarity of the PAO and MAB models, while the feasibility of these methods for PRB-SCF needs to be further investigated because diffuse functions are explicitly contained in the PRB.

In practice, however, even for systems as large as the L7 complexes, the Fock matrix construction step still dominates the computational cost of each SCF cycle due to its large prefactor, although asymptotically that step scales quadratically ${ }^{132}$ (or even lin-

\begin{tabular}{lcccc}
\hline & $N(\mathrm{MAB})$ & $N(\mathrm{aTZ})$ & $\lambda(\mathrm{MAB})$ & $\lambda(\mathrm{aTZ})$ \\
\hline C3A & 343 & 3473 & 12.17 & $5.07 \times 10^{12}$ \\
C3GC & 393 & 4002 & 12.57 & $7.93 \times 10^{12}$ \\
C2C2PD & 264 & 2760 & 13.50 & $4.32 \times 10^{11}$ \\
GCGC & 210 & 2208 & 9.02 & $1.39 \times 10^{8}$ \\
GGG & 180 & 1863 & 8.55 & $1.25 \times 10^{8}$ \\
CBH & 256 & 3404 & 15.63 & $1.45 \times 10^{9}$ \\
PHE & 267 & 3036 & 13.87 & $6.24 \times 10^{8}$ \\
\hline
\end{tabular}

TABLE XI: Comparison of sizes $(N)$ and overlap matrix condition numbers $(\lambda)$ of the MAB and the target aug-cc-pVTZ basis set on the L7 complexes.

early with special algorithms) $)^{39-45}$ with respect to system size. In our pilot implementation, $\mathbf{F}$ is built upon $\mathrm{PRB}$ or MAB density matrix projected into the secondary basis, which costs almost the same as that in a conventional SCF calculation within the same large basis. Therefore, to speed up these Fock build steps by taking advantage of the properties of the PRB and MAB becomes the most urgent task for MAB-SCF (PC) to outperform conventional $\mathrm{SCF}$ in terms of computational efficiency, especially for medium-sized systems. PRB-SCF can be reformulated as a conventional SCF calculation within a basis set whose size and shell structure are identical to $6-31+\mathrm{G}(\mathrm{d})$, due to the elimination of high angular momentum functions during the projection procedure, and the cost of the involved Fock builds thereby can be significantly reduced without using many other techniques. For MAB-SCF, instead of using Eq. 28, the MAB Fock matrix can be constructed by forming the ERI tensor in the primary basis first then contracting with the MAB density, as

$$
\begin{aligned}
\left(\mathcal{F}_{\alpha}\right)_{\alpha \beta}= & h_{\alpha \beta}+(\alpha \beta \mid \gamma \delta) \mathcal{P}_{\text {tot }}^{\gamma \delta}-\kappa(\alpha \gamma \mid \beta \delta) \mathcal{P}_{\alpha}^{\gamma \delta} \\
& +\left[V_{x c}\left(\mathcal{P}_{\alpha}, \mathcal{P}_{\beta}\right)\right]_{\alpha \beta} .
\end{aligned}
$$

And it is promising to us that quantities like $(\alpha \beta \mid \gamma \delta)$ can be efficiently computed (and stored) using the RI approximation, due to the highly compact and atom-blocked structure of the MAB. An algorithm that targets the fast evaluation of the Fock matrix in the context of SCF-MI (where the MO coefficients are block-diagonal) is under development in the Head-Gordon group recently, which can be readily adjusted for the demands here. An optimized implementation of MAB-SCF (PC) and the resulting timings vs. conventional SCF will be presented by a forthcoming paper. 


\section{CONCLUSION}

In this work, we proposed a new adaptive basis SCF method that can be used in KS-DFT calculations. The key aspects of this paper can be summarized as follows:

- The search for the MAB is based on an inexpensively obtained description of the electronic structure provided by PRB-SCF, and the variational minimization of the energy-like MAB objective function. Compared to the PAO model, MAB generates similar total energies and even closer relative energies, while the adaptive basis optimization procedure is decoupled with SCF iterations and encounters less convergence issues in general. Therefore, the MAB model can serve as a more tractable replacement of the PAO model in real applications.

- A preconditioned L-BFGS algorithm that requires the gradient and the on-diagonal blocks of the Hessian of the objective function is implemented to solve the MAB optimization problem; also, an approach that flexibly determines the MAB dimension based on the chemical environment is proposed. These can be adopted by PAO and other adaptive basis models as well.

- Perturbation corrections are applied on top of the MAB-SCF solutions to approach the desired accuracy, which resembles dual-basis SCF approaches but has no need to select or develop the paired basis subset. Instead, with the reference basis unchanged, the performance of MAB-SCF (PC) using different secondary basis sets is investigated on the G2 set, and the best accuracy is achieved with basis sets that are close to the CBS limit. The preferable correction scheme depends on the functional type. Based on the preliminary tests on the G2 set, PT2 demonstrates its effectiveness for pure functionals (GGAs, meta-GGAs), while DFPC is currently suggested for hybrid functionals.

- To demonstrate the versatile usefulness, the accuracy of MAB-SCF (PC) is assessed on G2 and many other $\mathrm{TC}$ and $\mathrm{NC}$ datasets. Compared to the exact SCF results, MAB-SCF (PC) generates $<0.15 \mathrm{kcal} / \mathrm{mol} \mathrm{RMSDs}$ for most of the TCs, and even smaller errors (usually $<0.1 \mathrm{kcal} / \mathrm{mol}$ ) for the NCs. Meanwhile, the functional RMSDs on several representative datasets (G2, W4-11, S22) are reproduced by MAB-SCF (PC) with only minimal differences from the conventional SCF results.

- Future work includes further refining the MABSCF (PC) model itself and its efficient implementation, as discussed in Sec.V. Based on its accuracy and the computational advantage of using an atom-centered minimal basis, MAB-SCF (PC) can be a promising substitute for conventional SCF to approach the basis set limit performance of modern density functionals with a more tractable cost. We hope to report the further progress on this research in due course.

\section{ACKNOWLEDGMENTS}

This work was partially supported by the grant "SI2-CHE: Development and Deployment of Chemical Software for Advanced Potential Energy Surfaces" from the U.S. National Science Foundation. We would like to thank Dr. Chris-Kriton Skylaris for helpful discussions on adaptive basis methods. Y. M. would like to thank Narbe Mardirossian for providing the datasets (input geometries and reference values) and the discussions on numerical tests.

\section{Appendix A: More details about the gradient of the MAB objective function}

Using the same notations as in Sec. II B, the most general form of the gradient of the MAB objective function is given by

$$
\frac{\partial E}{\partial \Delta^{Z p Z q}}=\sum_{X, Y} \frac{\partial R^{X \mu Y \nu}}{\partial \Delta^{Z p Z q}} G_{Y \nu X \mu},
$$

where $\mathbf{G}=$ SPSRF + FRSPS. With the parameterization of $\mathbf{B}$ by equations (12) and (13), 


$$
\begin{aligned}
& \frac{\partial R^{X \mu Y \nu}}{\partial \Delta^{Z p Z q}}=\left[C_{X p}^{X \mu} \delta_{Z}^{X} \sigma_{X q X i}-C_{X q}^{X \mu} \delta_{Z}^{X} \sigma_{X p X i}\right]\left(\sigma^{-1}\right)^{X i Y j}\left(B^{T}\right)_{Y j}^{Y \nu} \\
& +B_{X i}^{X \mu}\left(\sigma^{-1}\right)^{X i Y j}\left[C_{Y p}^{Y \nu} \delta_{Z}^{Y} \sigma_{Y q Y j}-C_{Y q}^{Y \nu} \delta_{Z}^{Y} \sigma_{Y p Y j}\right] \\
& -\sum_{W}(R S)_{W \sigma}^{X \mu}\left[C_{W p}^{W \sigma} \delta_{Z}^{W} \sigma_{W q W l}-C_{W q}^{W \sigma} \delta_{Z}^{W} \sigma_{W p W l}\right]\left(\sigma^{-1}\right)^{W l Y j}\left(B^{T}\right)_{Y j}^{Y \nu} \\
& -\sum_{W} B_{X i}^{X \mu}\left(\sigma^{-1}\right)^{X i W l}\left[C_{W p}^{W \sigma} \delta_{Z}^{W} \sigma_{W q W l}-C_{W q}^{W \sigma} \delta_{Z}^{W} \sigma_{W p W l}\right](S R)_{W \sigma}^{Y_{\nu}} .
\end{aligned}
$$

and correspondingly the gradient becomes

$$
\begin{aligned}
\frac{\partial E}{\partial \Delta^{Z_{p Z q}}}= & -2 \sigma_{Z_{p Z_{j}}}\left[\left(\sigma^{-1}\right) B^{T} G(I-R S) C\right]_{Z_{q}}^{Z_{j}} \\
& +2 \sigma_{Z_{q} Z_{j}}\left[\left(\sigma^{-1}\right) B^{T} G(I-R S) C\right]_{Z_{p}}^{Z_{j}}
\end{aligned}
$$

When $\boldsymbol{\Delta}$ stands for the orbital rotations within the MAB space, i.e., $p=i, q=j$, matrix $\mathbf{C}$ reduces to $\mathbf{B}$, and the gradient vanishes because evidently we have $(\mathbf{I}-\mathbf{R S}) \mathbf{B}=\mathbf{0}$. On the other hand, orbital rotations within the complementary space of the MAB ( $p=a, q=b$ ) have no effect on the objective function value either, simply due to the enforced on-atom orthogonality $\left(\sigma_{Z_{a} Z_{j}}=0\right)$. Therefore, the only non-zero block of this gradient is resulted from the rotations between these two subspaces. If we set $p=i, q=a$, only the first term in Eq. (A3) remains based on the arguments above, which leads to the gradient represented by Eq. (17).

\section{Appendix B: The preconditioned L-BFGS algorithm}

The basic idea of L-BFGS is to construct the approximate Hessian (inverse Hessian in practice) for the current iteration with gradients and steps computed in the most recent $m$ steps, where $m$ is the user-defined subspace size (number of "memorized" steps). If we define the gradient and displacement at $k$ th iteration as $\mathbf{g}_{\mathbf{k}}$ and $\mathbf{s}_{\mathbf{k}}$, and $\mathbf{y}_{\mathbf{k}}=\mathbf{g}_{\mathbf{k}+\mathbf{1}}-\mathbf{g}_{\mathbf{k}}$, the $k$ th approximate inverse Hessian can be evaluated as ${ }^{106}$ :

$$
\begin{aligned}
& \mathbf{H}_{\mathbf{k}}=\left(\mathbf{V}_{\mathbf{k}-\mathbf{1}}^{\mathbf{T}} \cdots \mathbf{V}_{\mathbf{k}-\mathbf{m}}^{\mathbf{T}}\right) \mathbf{H}_{\mathbf{k}}^{\mathbf{0}}\left(\mathbf{V}_{\mathbf{k}-\mathbf{m}} \cdots \mathbf{V}_{\mathbf{k}-\mathbf{1}}\right) \\
& +\rho_{\mathbf{k}-\mathbf{m}}\left(\mathbf{V}_{\mathbf{k}-\mathbf{1}}^{\mathbf{T}} \cdots \mathbf{V}_{\mathbf{k}-\mathbf{m}+\mathbf{1}}^{\mathbf{T}}\right) \mathbf{s}_{\mathbf{k}-\mathbf{m}} \mathbf{s}_{\mathbf{k}-\mathbf{m}}^{\mathbf{T}}\left(\mathbf{V}_{\mathbf{k}-\mathbf{m}+\mathbf{1}} \cdots \mathbf{V}_{\mathbf{k}-\mathbf{1}}\right) \\
& +\rho_{\mathrm{k}-\mathbf{m}+\mathbf{1}}\left(\mathbf{V}_{\mathbf{k}-\mathbf{1}}^{\mathbf{T}} \cdots \mathbf{V}_{\mathbf{k}-\mathbf{m}+\mathbf{2}}^{\mathbf{T}}\right) \mathbf{s}_{\mathbf{k}-\mathbf{m}+\mathbf{1}} \mathbf{s}_{\mathbf{k}-\mathbf{m}+\mathbf{1}}^{\mathbf{T}}\left(\mathbf{V}_{\mathbf{k}-\mathbf{m}+\mathbf{2}} \cdots \mathbf{V}_{\mathbf{k}-\mathbf{1}}\right) \\
& +\cdots+\rho_{\mathrm{k}-1} \mathbf{s}_{\mathrm{k}-1} \mathbf{s}_{\mathrm{k}-1}^{\mathrm{T}} \text {. }
\end{aligned}
$$

where

$$
\rho_{\mathbf{k}}=\frac{1}{\mathbf{y}_{\mathbf{k}}^{\mathbf{T}} \mathbf{s}_{\mathbf{k}}}, \quad \mathbf{V}_{\mathbf{k}}=\mathbf{I}-\rho_{\mathbf{k}} \mathbf{y}_{\mathbf{k}} \mathbf{s}_{\mathbf{k}}^{\mathbf{T}} .
$$

In practice, a "two-loop" algorithm which only requires vector-vector products is implemented to compute $\mathbf{H}_{\mathbf{k}}$ acting on $\mathbf{g}_{\mathbf{k}}$. $\mathbf{H}_{\mathbf{k}}^{\mathbf{0}}$ is the preconditioner for the approximate inverse Hessian. By default, a constant scaling factor is used for $\mathbf{H}_{\mathbf{k}}^{\mathbf{0}}$, which is considered as the unpreconditioned case here. Once $\mathbf{H}_{\mathbf{k}}^{\mathbf{0}}$ contains more information about the true inverse Hessian, the step generated by L-BFGS be- comes closer to a Newton step (more efficient descending) from the current position, which accelerates the convergence.

If we still denote $\mathbf{G}=\mathbf{S P S R F}+\mathbf{F R S P S}$, the Hessian of the MAB objective function (Eq. (8)) can be formally represented as follows (only on-block 
mixings are allowed):

$$
\begin{aligned}
& H_{X_{i} X_{a}, Y_{j} Y_{b}}=\left.\frac{\partial^{2} E}{\partial \Delta^{X_{i} X_{a}} \partial \Delta^{Y_{j} Y_{b}}}\right|_{\Delta=0} \\
= & \mathbf{R}^{\boldsymbol{\Delta}} \mathbf{S P S R}^{\boldsymbol{\Delta}} \mathbf{F}+\mathbf{R}^{\boldsymbol{\Delta} \boldsymbol{\Delta}} \mathbf{G} \\
= & 2\left(P_{o v}\right)_{X_{i} Y_{b}}\left(F_{o v}\right)_{Y_{j} X_{a}}+2\left(F_{o v}\right)_{X_{i} Y_{b}}\left(P_{o v}\right)_{Y_{j} X_{a}} \\
+ & 2\left(P_{o o}\right)_{X_{i} Y_{j}}\left(F_{v v}\right)_{Y_{b} X_{a}}+2\left(F_{o o}\right)_{X_{i} Y_{j}}\left(P_{v v}\right)_{Y_{b} X_{a}} \\
- & 2\left(S_{o v}\right)_{X_{i} Y_{b}}\left(G_{o v}\right)_{Y_{j} X_{a}}-2\left(G_{o v}\right)_{X_{i} Y_{b}}\left(S_{o v}\right)_{Y_{j} X_{a}} \\
+ & 2\left(S_{o o}\right)_{X_{i} Y_{j}}\left(G_{v v}\right)_{Y_{b} X_{a}}-2\left(G_{o o}\right)_{X_{i} Y_{j}}\left(S_{v v}\right)_{Y_{b} X_{a}},
\end{aligned}
$$

where $\mathbf{R}^{\boldsymbol{\Delta}}$ and $\mathbf{R}^{\boldsymbol{\Delta} \boldsymbol{\Delta}}$ stands for first- and secondorder derivatives of $\mathbf{R}$ with respect to $\boldsymbol{\Delta}$. The first four terms in the result of Eq. (B3) come from the $\mathbf{R}^{\boldsymbol{\Delta}} \mathbf{R}^{\boldsymbol{\Delta}}$ term, while the rest from the $\mathbf{R}^{\boldsymbol{\Delta} \boldsymbol{\Delta}}$ term. The explicit forms of the involved matrix elements are:

$$
\begin{aligned}
\left(P_{O o}\right)_{X i Y j} & =\sigma_{X i X k}\left[\sigma^{-1} B^{T} S P S B \sigma^{-1}\right]^{X k Y l} \sigma_{Y l Y j} \\
\left(P_{o v}\right)_{X i Y a} & =\sigma_{X i X j}\left[\sigma^{-1} B^{T} S P S(I-R S)\right]_{Y \nu}^{X j} V_{Y a}^{Y \nu} \\
\left(P_{v v}\right)_{X a Y b} & =\left(V^{T}\right)_{X a}^{X \mu}[(I-S R) S P S(I-R S)]_{X \mu Y \nu} V_{Y b}^{Y \nu},
\end{aligned}
$$

$$
\begin{aligned}
\left(F_{O o}\right)_{X i Y j} & =\sigma_{X i X k}\left[\sigma^{-1} B^{T} F B \sigma^{-1}\right]^{X k Y l} \sigma_{Y l Y j} \\
\left(F_{o v}\right)_{X i Y a} & =\sigma_{X i X j}\left[\sigma^{-1} B^{T} F(I-R S)\right]_{Y \nu}^{X j} V_{Y Y^{Y}}^{Y} \\
\left(F_{v v}\right)_{X a Y b} & =\left(V^{T}\right)_{X a}^{X \mu}[(I-S R) F(I-R S)]_{X \mu Y \nu} V_{Y \nu}^{Y \nu},
\end{aligned}
$$

$$
\begin{aligned}
\left(G_{O o}\right)_{X i Y j} & =\sigma_{X i X k}\left[\sigma^{-1} B^{T} G B \sigma^{-1}\right]^{X k Y l} \sigma_{Y l Y j} \\
\left(G_{O v}\right)_{X i Y a} & =\sigma_{X i X j}\left[\sigma^{-1} B^{T} G(I-R S)\right]_{Y \nu}^{X j} V_{Y Y^{Y}}^{Y} \\
\left(G_{v v}\right)_{X a Y b} & =\left(V^{T}\right)_{X a}^{X \mu}[(I-S R) G(I-R S)]_{X \mu Y \nu} V_{Y \nu}^{Y \nu},
\end{aligned}
$$

and

$$
\begin{aligned}
\left(S_{o o}\right)_{X i Y j} & =\sigma_{X i X k}\left(\sigma^{-1}\right)^{X k Y l} \sigma_{Y l Y j} \\
\left(S_{v v}\right)_{X a Y b} & =\left(V^{T}\right)_{X a}^{X \mu}[S(I-R S)]_{X \mu Y \nu} V_{Y b}^{Y \nu} \\
\left(S_{O v}\right)_{X i Y a} & =\sigma_{X i X j}\left[\left(\sigma^{-1}\right) B^{T} S\right]_{Y j}^{X j} V_{Y a}^{Y \nu},
\end{aligned}
$$

and in fact $\sigma_{X i X j}=\delta_{i j}$ when on-block orthogonality is enforced. More details about the Hessian derivation (especially the $\mathbf{R}^{\Delta \Delta}$ term) can be found in Ref. 133, which carefully derived the orbital rotation Hessian for SCF-MI. The preconditioner we apply to the L-BFGS algorithm is the inverted ondiagonal blocks of the Hessian, i.e., the inverse of
$\mathbf{H}_{\mathbf{x x}}$ for all the different atom blocks $(X)$. Within the "two-loop" implementation, $\mathbf{H}_{\mathbf{k}}^{\mathbf{0}}$ acts on vector $\mathbf{v}=\mathbf{V}_{\mathbf{k}-\mathbf{m}} \mathbf{V}_{\mathbf{k}-\mathbf{m}+\mathbf{1}} \cdots \mathbf{V}_{\mathbf{k}-\mathbf{1}} \mathbf{g}_{\mathbf{k}}$, which can be segmented into contributions from each atom block. Therefore, the application of the preconditioner is equivalent to solving the following linear equation on each atom block:

$$
H_{X i X a, X j X b} u^{X j X b}=v_{X i X a},
$$

where $\mathbf{u}$ is the preconditioned vector: $\mathbf{u}=$ $\mathbf{H}_{k}^{0} \mathbf{v}$. Based on the property of the Hessian matrix (symmetric positive-definite), a preconditioned conjugate-gradient $(\mathrm{CG})$ algorithm is implemented to solve Eq. (B8) iteratively on each atom block. The implemented preconditioner for CG is actually the inverse of the on-diagonal part $(X=Y)$ of the last two terms in Eq. (B3).

\section{Appendix C: Construction of pseudo-canonicalized MOs upon MAB-SCF solution}

Once MAB-SCF converges, a Fock matrix in the secondary basis can be built upon the MAB density matrix projected into the secondary basis:

$$
\mathbf{F}=\mathbf{F}(\tilde{\mathbf{P}}), \quad \tilde{\mathbf{P}}=\mathbf{B} \mathcal{P} \mathbf{B}^{T} .
$$

To form the pseudo-canonicalized occupied and virtual MOs, in theory we need to diagonalize $\mathbf{F}_{O O}$ and $\mathbf{F}_{V V}$ separately. In fact, the occupied ones are already available in this case, since we can simply project the occupied MOs optimized by MAB-SCF into the large basis: $\left(C_{o}\right)_{i}^{\mu}=B_{\alpha}^{\mu}\left(\mathcal{C}_{o}\right)_{i}^{\alpha}$. Obviously, $\mathbf{C}_{o}$ diagonalizes $\mathbf{F}$ :

$$
\left(C_{o}^{T}\right)_{i}{ }^{\mu} F_{\mu \nu}\left(C_{o}\right)^{\nu}{ }_{j}=\left(\mathcal{C}_{o}^{T}\right)_{i}{ }^{\alpha} F_{\alpha \beta}\left(\mathcal{C}_{o}\right)^{\beta}{ }_{j}=\epsilon_{i} \delta_{i j} .
$$

To obtain the eigenvalues and eigenvectors of $\mathbf{F}_{V V}$, we first form an orthonormal basis that spans the virtual space. If the full but non-redundant span of the secondary basis is represented by $\mathbf{X}\left(\mathbf{X}^{T} \mathbf{S X}=\right.$ $\mathbf{I})$, the demanded vectors can be generated by projecting out the space spanned by occupied MOs:

$$
\mathbf{V}=(\mathbf{I}-\tilde{\mathbf{P}} \mathbf{S}) \mathbf{X} \text {. }
$$

The vectors in $\mathbf{V}$ can be orthonormalized again by performing a canonical orthogonalization (diagonalizing $\mathbf{V}^{T} \mathbf{S V}$ will be required). Also, after doing this, the linear dependency of vectors in $\mathbf{V}$ will be eliminated and its column dimension reduces to $N_{v}$. We denote the resulting orthonormal basis as $\mathbf{V}^{\prime}$. Solving the following standard eigenvalue problem

$$
\left(\mathbf{V}^{\prime T} \mathbf{F} \mathbf{V}^{\prime}\right) \mathbf{C}_{v}^{\prime}=\boldsymbol{\epsilon}_{v} \mathbf{C}_{v}^{\prime},
$$


the energies of the pseudo-canonicalized virtual orbitals ( $\epsilon_{a}$ 's in Eq. 27) are given by $\boldsymbol{\epsilon}_{v}$, and their coefficients $\mathbf{C}_{v}=\mathbf{V}^{\prime} \mathbf{C}_{v}^{\prime}$. The Fock matrix elements coupling between occupied and virtual pseudocanonicalized MOs $\left(\mathbf{F}_{O V}\right)$ can be evaluated as

$$
F_{i a}^{(1)}=\left(\mathcal{C}_{o}^{T}\right)_{i}^{\alpha}\left(B^{T}\right)_{\alpha}^{\mu} F_{\mu \nu}\left(C_{v}\right)_{a}^{\nu}
$$

${ }^{1}$ P. Hohenberg and W. Kohn, Phys. Rev. 136, B864 (1964). ${ }^{2}$ W. Kohn and L. J. Sham, Phys. Rev. 140, 1133 (1965).

${ }^{3}$ W. Kohn, A. D. Becke, and R. G. Parr, J. Phys. Chem. 100, 12974 (1996).

${ }^{4}$ A. D. Becke, J. Chem. Phys. 84, 4524 (1986).

${ }^{5}$ J. P. Perdew, K. Burke, and M. Ernzerhof, Phys. Rev. Lett. 77, 3865 (1996).

${ }^{6}$ A. D. Becke, J. Chem. Phys. 109, 2092 (1998).

${ }^{7}$ J. P. Perdew, S. Kurth, A. Zupan, and P. Blaha, Phys. Rev. Lett. 82, 2544 (1999).

${ }^{8}$ J. Tao, J. P. Perdew, V. N. Staroverov, and G. E. Scuseria, Phys. Rev. Lett. 91, 146401 (2003).

${ }^{9}$ A. D. Becke, J. Chem. Phys. 98, 5648 (1993).

${ }^{10}$ T. Leininger, H. Stoll, H.-J. Werner, and A. Savin, Chem. Phys. Lett. 275, 151 (1997).

${ }^{11}$ H. Iikura, T. Tsuneda, T. Yanai, and K. Hirao, J. Chem. Phys. 115, 3540 (2001).

${ }^{12}$ J. Klimeš and A. Michaelides, J. Chem. Phys. 137, 120901 (2012).

${ }^{13}$ S. Grimme, J. Comput. Chem. 25, 1463 (2004).

${ }^{14}$ S. Grimme, J. Comput. Chem. 27, 1787 (2006).

${ }^{15}$ S. Grimme, J. Antony, S. Ehrlich, and H. Krieg, J. Chem. Phys. 132, 154104 (2010).

${ }^{16}$ K. Lee, É. D. Murray, L. Kong, B. I. Lundqvist, and D. C. Langreth, Phys. Rev. B 82, 081101 (2010).

${ }^{17}$ O. A. Vydrov and T. van Voorhis, J. Chem. Phys. 133, 244103 (2010).

${ }^{18}$ N. Mardirossian and M. Head-Gordon, Phys. Chem. Chem. Phys. 16, 9904 (2014).

${ }^{19}$ N. Mardirossian and M. Head-Gordon, J. Chem. Phys. 142, 074111 (2015).

${ }^{20}$ J. A. Pople, P. M. Gill, and B. G. Johnson, Chem. Phys. Lett. 199, 557 (1992).

${ }^{21}$ K. S. Raymond and R. A. Wheeler, J. Comput. Chem. 20, 207 (1999).

${ }^{22}$ A. D. Boese, J. M. Martin, and N. C. Handy, J. Chem. Phys. 119, 3005 (2003).

${ }^{23}$ N. X. Wang and A. K. Wilson, J. Chem. Phys. 121, 7632 (2004).

${ }^{24}$ N. X. Wang and A. K. Wilson, Mol. Phys. 103, 345 (2005).

${ }^{25}$ A. D. Becke, Phys. Rev. A 38, 3098 (1988).

${ }^{26}$ C. Lee, W. Yang, and R. G. Parr, Phys. Rev. B 37, 785 (1988).

${ }^{27}$ T. H. Dunning Jr, J. Chem. Phys. 90, 1007 (1989).

${ }^{28}$ D. E. Woon and T. H. Dunning Jr, J. Chem. Phys. 98, 1358 (1993).

${ }^{29}$ J. Rezac and P. Hobza, J. Chem. Theory. Comput. 9, 2151 (2013).

${ }^{30}$ J. Rezac, K. E. Riley, and P. Hobza, J. Chem. Theory. Comput. 7, 2427 (2011).

${ }^{31}$ J. Rezac, K. E. Riley, and P. Hobza, J. Chem. Theory. Comput. 7, 3466 (2011).

${ }^{32}$ D. Rappoport and F. Furche, J. Chem. Phys. 133, 134105 (2010).
${ }^{33}$ F. B. Van Duijneveldt, J. G. van Duijneveldt-van de Rijdt, and J. H. van Lenthe, Chem. Rev. 94, 1873 (1994).

${ }^{34}$ L. A. Curtiss, K. Raghavachari, P. C. Redfern, and J. A. Pople, J. Chem. Phys. 106, 1063 (1997).

${ }^{35}$ R. Haunschild and W. Klopper, J. Chem. Phys. 136, 164102 (2012).

${ }^{36}$ R. D. Adamson, P. M. Gill, and J. A. Pople, Chem. Phys. Lett. 284, 6 (1998).

${ }^{37}$ S. Grimme, J. G. Brandenburg, C. Bannwarth, and A. Hansen, J. Chem. Phys. 143, 054107 (2015).

${ }^{38}$ Y. Zhao and D. G. Truhlar, Theor. Chem. Acc. 120, 215 (2008).

${ }^{39}$ C. A. White, B. G. Johnson, P. M. Gill, and M. HeadGordon, Chem. Phys. Lett. 230, 8 (1994).

${ }^{40}$ C. A. White, B. G. Johnson, P. M. Gill, and M. HeadGordon, Chem. Phys. Lett. 253, 268 (1996).

${ }^{41}$ C. A. White and M. Head-Gordon, J. Chem. Phys. 104, 2620 (1996).

${ }^{42}$ M. C. Strain, G. E. Scuseria, and M. J. Frisch, Science 271, 51 (1996).

${ }^{43}$ C. Ochsenfeld, C. A. White, and M. Head-Gordon, J. Chem. Phys. 109, 1663 (1998).

${ }^{44}$ G. Lippert, J. Hutter, and M. Parrinello, Theor. Chem. Acc. 103, 124 (1999).

${ }^{45}$ J. Dziedzic, Q. Hill, and C.-K. Skylaris, J. Chem. Phys. 139, 214103 (2013).

${ }^{46}$ S. Goedecker, Rev. Mod. Phys. 71, 1085 (1999).

${ }^{47}$ D. Bowler and T. Miyazaki, Rep. Prog. Phys. 75, 036503 (2012).

${ }^{48}$ P. E. Maslen, C. Ochsenfeld, C. A. White, M. S. Lee, and M. Head-Gordon, J. Phys. Chem. A 102, 2215 (1998).

${ }^{49}$ G. Berghold, M. Parrinello, and J. Hutter, J. Chem. Phys. 116, 1800 (2002).

${ }^{50}$ K. Eichkorn, O. Treutler, H. Öhm, M. Häser, and R. Ahlrichs, Chem. Phys. Lett. 240, 283 (1995).

${ }^{51}$ H. A. Früchtl, R. A. Kendall, R. J. Harrison, and K. G. Dyall, Int. J. Quantum Chem. 64, 63 (1997).

${ }^{52}$ F. Weigend, Phys. Chem. Chem. Phys. 4, 4285 (2002).

${ }^{53}$ A. Sodt, J. E. Subotnik, and M. Head-Gordon, J. Chem. Phys. 125, 194109 (2006).

${ }^{54}$ A. Sodt and M. Head-Gordon, J. Chem. Phys. 128, 104106 (2008).

${ }^{55}$ M. Sierka, A. Hogekamp, and R. Ahlrichs, J. Chem. Phys. 118, 9136 (2003).

${ }^{56}$ S. Manzer, P. R. Horn, N. Mardirossian, and M. HeadGordon, J. Chem. Phys. 143, 024113 (2015).

${ }^{57}$ R. Jurgens-Lutovsky and J. Almlöf, Chem. Phys. Lett. 178, 451 (1991).

${ }^{58}$ K. Wolinski and P. Pulay, J. Chem. Phys. 118, 9497 (2003).

${ }^{59}$ W. Liang and M. Head-Gordon, J. Phys. Chem. A 108, 3206 (2004).

${ }^{60}$ R. P. Steele, R. A. DiStasio Jr, Y. Shao, J. Kong, and M. Head-Gordon, J. Chem. Phys. 125, 074108 (2006).

${ }^{61}$ R. P. Steele, Y. Shao, R. A. DiStasio, and M. HeadGordon, J. Phys. Chem. A 110, 13915 (2006).

${ }^{62}$ R. P. Steele and M. Head-Gordon, Mol. Phys. 105, 2455 (2007).

${ }^{63}$ R. P. Steele, R. A. DiStasio Jr, and M. Head-Gordon, J. Chem. Theory. Comput. 5, 1560 (2009).

${ }^{64}$ J. Deng, A. T. Gilbert, and P. M. Gill, J. Chem. Phys. 130, 231101 (2009).

${ }^{65}$ J. Deng, A. T. Gilbert, and P. M. Gill, J. Chem. Phys. 133, 044116 (2010). 
${ }^{66}$ J. Deng, A. T. Gilbert, and P. M. Gill, Phys. Chem. Chem. Phys. 12, 10759 (2010).

${ }^{67}$ R. Mulliken, J. Chem. Phys. 36, 3428 (1962).

${ }^{68}$ E. R. Davidson, J. Chem. Phys. 46, 3320 (1967).

${ }^{69}$ K. R. Roby, Mol. Phys. 27, 81 (1974).

${ }^{70}$ R. Heinzmann and R. Ahlrichs, Theor. Chim. Acta. 42, 33 (1976)

${ }^{71}$ C. Ehrhardt and R. Ahlrichs, Theor. Chim. Acta. 68, 231 (1985).

72 A. E. Reed, L. A. Curtiss, and F. Weinhold, Chem. Rev. 88, 899 (1988).

${ }^{73}$ M. S. Lee and M. Head-Gordon, Int. J. Quantum Chem. 76, 169 (2000).

${ }^{74}$ I. Mayer, Can. J. Chem. 74, 939 (1996).

${ }^{75}$ J. Cioslowski and A. Liashenko, J. Chem. Phys. 108, 4405 (1998).

${ }^{76}$ W. Lu, C. Wang, M. Schmidt, L. Bytautas, K. Ho, and K. Ruedenberg, J. Chem. Phys. 120, 2629 (2004).

${ }^{77}$ D. N. Laikov, Int. J. Quantum Chem. 111, 2851 (2011).

${ }^{78}$ G. Knizia, J. Chem. Theory. Comput. 9, 4834 (2013).

${ }^{79}$ W. H. Adams, J. Chem. Phys. 34, 89 (1961).

${ }^{80}$ W. H. Adams, J. Chem. Phys. 37, 2009 (1962).

${ }^{81}$ H. Stoll, G. Wagenblast, and H. Preu $\beta$, Theor. Chim. Acta. 57, 169 (1980).

${ }^{82}$ R. Z. Khaliullin, M. Head-Gordon, and A. T. Bell, J. Chem. Phys. 124, 204105 (2006).

${ }^{83}$ P. R. Horn, E. J. Sundstrom, T. A. Baker, and M. HeadGordon, J. Chem. Phys. 138, 134119 (2013).

${ }^{84}$ W. Adams, Chem. Phys. Lett. 12, 295 (1971).

${ }^{85}$ M. S. Lee and M. Head-Gordon, J. Chem. Phys. 107, 9085 (1997).

${ }^{86}$ M. S. Lee and M. Head-Gordon, Computers \& chemistry 24, 295 (2000).

${ }^{87}$ T. Ozaki, Phys. Rev. B 67, 155108 (2003).

${ }^{88}$ T. Ozaki and H. Kino, Phys. Rev. B 69, 195113 (2004).

${ }^{89}$ J. Junquera, Ó. Paz, D. Sánchez-Portal, and E. Artacho, Phys. Rev. B 64, 235111 (2001).

${ }^{90}$ M. Basanta, Y. Dappe, P. Jelínek, and J. Ortega, Comput. Mater. Sci. 39, 759 (2007).

${ }^{91}$ D. Bowler and T. Miyazaki, J. Phys.: Condens. Matter 22, 074207 (2010).

${ }^{92}$ D. Bowler, T. Miyazaki, and M. Gillan, J. Phys.: Condens. Matter 14, 2781 (2002).

${ }^{93}$ A. Torralba, M. Todorović, V. Brázdová, R. Choudhury, T. Miyazaki, M. Gillan, and D. Bowler, J. Phys.: Condens. Matter 20, 294206 (2008).

${ }^{94}$ C.-K. Skylaris, P. D. Haynes, A. A. Mostofi, and M. C. Payne, J. Chem. Phys. 122, 084119 (2005).

${ }^{95}$ C.-K. Skylaris, P. D. Haynes, A. A. Mostofi, and M. C. Payne, J. Phys.: Condens. Matter 20, 064209 (2008).

${ }^{96}$ C.-K. Skylaris, A. A. Mostofi, P. D. Haynes, O. Diéguez, and M. C. Payne, Phys. Rev. B 66, 035119 (2002).

${ }^{97}$ A. A. Mostofi, P. D. Haynes, C.-K. Skylaris, and M. C. Payne, J. Chem. Phys. 119, 8842 (2003).

${ }^{98}$ M. Rayson and P. Briddon, Phys. Rev. B 80, 205104 (2009).

${ }^{99}$ M. Rayson, Comput. Phys. Commun. 181, 1051 (2010).

${ }^{100}$ A. Nakata, D. R. Bowler, and T. Miyazaki, J. Chem. Theory. Comput. 10, 4813 (2014).

${ }^{101}$ L. Lin, J. Lu, L. Ying, and E. Weinan, J. Comput. Phys. 231, 2140 (2012).

${ }^{102}$ L. Lin, J. Lu, L. Ying, and E. Weinan, J. Comput. Phys. 231, 4515 (2012).

${ }^{103}$ M. Head-Gordon, P. E. Maslen, and C. A. White, J. Chem. Phys. 108, 616 (1998).
${ }^{104}$ J. Hutter, M. Parrinello, and S. Vogel, J. Chem. Phys. 101, 3862 (1994).

${ }^{105}$ Y. Shao, Z. Gan, E. Epifanovsky, A. T. Gilbert, M. Wormit, J. Kussmann, A. W. Lange, A. Behn, J. Deng, X. Feng, et al., Mol. Phys. 113, 184 (2015).

${ }^{106} \mathrm{~J}$. Nocedal and S. Wright, Numerical optimization (Springer Science \& Business Media, 2006).

${ }^{107}$ D. C. Liu and J. Nocedal, Math. Program. 45, 503 (1989).

${ }^{108}$ P. M. Gill, B. G. Johnson, and J. A. Pople, Chem. Phys. Lett. 209, 506 (1993).

${ }^{109}$ T. Van Voorhis and M. Head-Gordon, Mol. Phys. 100, 1713 (2002).

${ }^{110}$ J. Sun, R. Haunschild, B. Xiao, I. W. Bulik, G. E. Scuseria, and J. P. Perdew, J. Chem. Phys. 138, 044113 (2013).

${ }^{111}$ Y. Zhao and D. G. Truhlar, J. Chem. Phys. 125, 194101 (2006).

${ }^{112}$ V. N. Staroverov, G. E. Scuseria, J. Tao, and J. P. Perdew, J. Chem. Phys. 119, 12129 (2003).

${ }^{113}$ C. Adamo and V. Barone, J. Chem. Phys. 110, 6158 (1999).

${ }^{114}$ J.-D. Chai and M. Head-Gordon, Phys. Chem. Chem. Phys. 10, 6615 (2008).

${ }^{115}$ F. Jensen, J. Chem. Phys. 115, 9113 (2001).

${ }^{116}$ F. Jensen, J. Chem. Phys. 116, 7372 (2002).

${ }^{117}$ F. Jensen, J. Chem. Phys. 117, 9234 (2002).

${ }^{118}$ R. Krishnan, J. S. Binkley, R. Seeger, and J. A. Pople, J. Chem. Phys. 72, 650 (1980).

${ }^{119}$ M. J. Frisch, J. A. Pople, and J. S. Binkley, J. Chem. Phys. 80, 3265 (1984).

${ }^{120}$ A. Karton, S. Daon, and J. M. Martin, Chem. Phys. Lett. 510, 165 (2011).

${ }^{121}$ L. A. Curtiss, K. Raghavachari, G. W. Trucks, and J. A. Pople, J. Chem. Phys. 94, 7221 (1991).

${ }^{122}$ L. Goerigk and S. Grimme, J. Chem. Theory. Comput. 7, 291 (2011).

${ }^{123}$ Y. Zhao, N. González-García, and D. G. Truhlar, J. Phys. Chem. A 109, 2012 (2005).

${ }^{124}$ Y. Zhao, B. J. Lynch, and D. G. Truhlar, Phys. Chem. Chem. Phys. 7, 43 (2005).

${ }^{125}$ J. M. Martin, J. Phys. Chem. A 117, 3118 (2013).

${ }^{126}$ P. Jurečka, J. Šponer, J. Černỳ, and P. Hobza, Phys. Chem. Chem. Phys. 8, 1985 (2006).

${ }^{127}$ M. S. Marshall, L. A. Burns, and C. D. Sherrill, J. Chem. Phys. 135, 194102 (2011).

${ }^{128}$ J. Rezac and P. Hobza, J. Chem. Theory. Comput. 8, 141 (2012).

${ }^{129}$ K. U. Lao and J. M. Herbert, J. Chem. Phys. 139, 034107 (2013).

${ }^{130}$ K. U. Lao and J. M. Herbert, J. Phys. Chem. A 119, 235 (2015).

${ }^{131}$ R. Sedlak, T. Janowski, M. Pitonak, J. Rezac, P. Pulay, and P. Hobza, J. Chem. Theory. Comput. 9, 3364 (2013).

${ }^{132}$ D. L. Strout and G. E. Scuseria, J. Chem. Phys. 102, 8448 (1995).

${ }^{133}$ P. R. Horn and M. Head-Gordon, J. Chem. Phys. 143, 114111 (2015). 